This is the final peer-reviewed accepted manuscript of:

S. CC, V. Raychoudhury, G. Marfia, e A. Singla, «A survey of routing and data dissemination in Delay Tolerant Networks", J. Netw. Comput. Appl., vol. 67, pagg. 128-146, mag. 2016, doi: 10.1016/j.jnca.2016.01.002.

The final published version is available online at:

https://doi.org/10.1016/j.jnca.2016.01.002

Rights / License:

The terms and conditions for the reuse of this version of the manuscript are specified in the publishing policy. For all terms of use and more information see the publisher's website.

This item was downloaded from IRIS Università di Bologna (https://cris.unibo.it/)

When citing, please refer to the published version. 


\title{
A Survey of Routing and Data Dissemination in Delay Tolerant Networks
}

\author{
Sobin $\mathrm{CC}^{1}$, Vaskar Raychoudhury ${ }^{1}$, Gustavo Marfia ${ }^{2}$, Ankita Singla ${ }^{1}$ \\ ${ }^{1}$ IIT Roorkee, India. E-mail: \{sobincc, ankitajnd\}@gmail.com,vaskar@ieee.org \\ ${ }^{2}$ University of Bologna, Italy. E-mail: gustavo.marfia@unibo.it
}

\begin{abstract}
Delay Tolerant Networks (DTNs) have practical applications in various fields. DTNs have been studied in-depth by many researchers and multiple high quality survey papers have been produced which analyzes DTN features, taxonomies, and applications. In recent years, interest in DTN research has rekindled as there are several emerging network-based application domains that require delay tolerance support and thus can use DTN specific routing and data dissemination techniques. Examples of such novel areas are Information Centric Network (ICN), Mobile ICN, Named Data Network (NDN), Internet of Things (IoT), etc. In this paper, we have surveyed those applications briefly and have proposed an alternate taxonomy for classifying existing DTN routing algorithms. The objective of this survey is to help future researchers to identify DTN specific properties in the new applications and to apply appropriate routing protocols whenever necessary. We have studied the relation between message replication and individual or group communication semantics of DTN routing protocols considering both social-based and opportunistic message forwarding techniques. We have also introduced an in-depth coverage of data dissemination protocols in DTN which can be adapted to content-centric networking domains. We conclude our survey by identifying a set of open challenges for future researchers.
\end{abstract}

Index Terms - Delay Tolerant Networks; Routing; Data Dissemination; MANET

\section{Introduction}

Delay tolerant networks (DTNs) or opportunistic networks [1] are sparse mobile networks in which an end-to-end path may not exist. The underlying principle behind DTN to route data is store-carry and forward, in which intermediate mobile nodes store data to be transmitted till it finds an appropriate relay node (to forward the message) in the path towards the destination. DTNs have applications in several ad hoc networking and data dissemination operations, like crisis management, battlefield, wildlife monitoring [2], transportation engineering [3], Pocket Switched Networks [4], etc. Recently, several emerging and novel network-based application domains are coming up that also require delay tolerance support. Examples of such novel areas are Mobile Information Centric Network (M-ICN), Named Data Network (NDN) (a class of ICN), Internet of Things (IoT), Mobile Social Networks (MoSoN), etc. In this paper, we have looked into DTN specific routing and data dissemination techniques which can be adapted for the aforementioned application domains.

DTN evolved from the Mobile Ad hoc Networks (MANET) by relaxing some of the requirements of MANET and by allowing a high degree of mobility to the participating nodes (Fig. 1). MANET and DTN share many common aspects, such as, lack of infrastructure, resource constrains, node mobility resulting in frequent network partitioning, etc. However, they have multiple differences as well. While MANET nodes communicate using standard TCP/IP protocol suite, DTN nodes use application-layer Bundle protocols, designed to support the store-carry-forward communication paradigm of DTN, to communicate with peers. Bundle layer protocol span across transport and network layers: storing limited bundles inside the network and facilitating late binding of DTN address to subnet address [5]. Network partition caused by node mobility results in packet loss in MANET whereas, it stretches the span of DTN as routing through frequent disconnection is an inherent property of DTN routing protocols. Finding the appropriate relay node may take long time in DTN which requires message carriers to store the message in their buffers for considerably long time.

Routing in DTNs is non-trivial due to the following challenges. Firstly, DTNs has no pre-existing infrastructure like wired and wireless infrastructure-based networks. Even infrastructure-less MANETs computes end-to-end routing paths which are, instead, inherently non-existent in DTNs. So, traditional MANET protocols like AODV [6], DSR [7], etc. cannot be directly applied to DTN because they require the existence of a fully connected graph to route a message and fail otherwise. Secondly, DTN nodes store messages until they find a relay node to forward that message towards the destination. Finding the relay node is crucial to DTN routing as it directly affects performance in terms of delay in message delivery and may even result in message loss. Due to the intermittent connectivity and long delay in communications, it is often difficult to develop an optimal forwarding strategy for routing in DTN.

DTNs have their own routing protocols, and several of them have been developed in the recent years. Existing DTN routing protocols (such as PROPHET [8]) have adopted either of the two types of forwarding strategies for relay selection - pure opportunistic or social-based. Pure opportunistic selection of relay nodes is based on factors like, probability of a 
node contacting the destination node, mobility patterns from encounter information of various nodes, historical contacts of a node with the destination, etc. Social-based relay selection uses various social metrics such as, betweenness centrality, community, etc. The selection of a routing strategy also depends on any available prior information regarding the topology of the network.

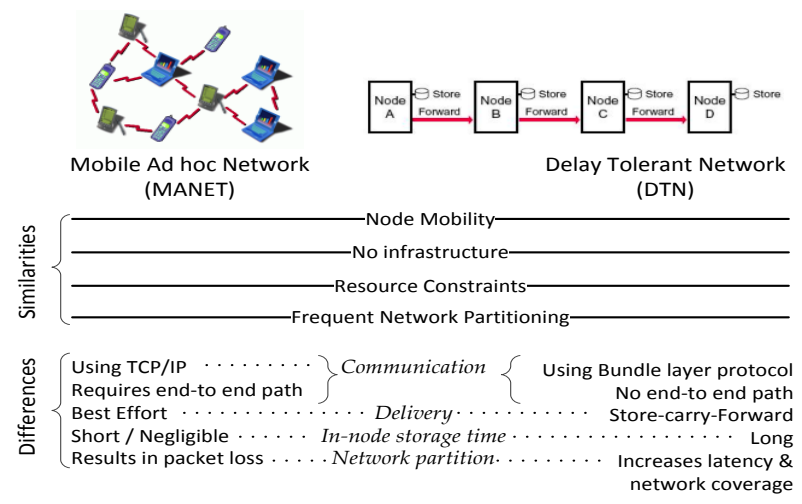

Fig. 1. Similarities and Differences of MANET and DTN

A number of high-quality survey papers have appeared in literature covering DTN routing protocols. We can grossly classify (Fig. 2) them into knowledge-based (depending on whether knowledge of future movements of nodes and resultant network topologies are available or not) [9], social-based (use social relations among the nodes for packet forwarding) [10][11], delivery semantics based (use network semantics, like unicast, multicast, or anycast for packet forwarding) [12] categories. There are also two other surveys [13][14] which focus mainly on design issues and challenges of DTN as a whole with a small coverage of some routing protocols without giving any proper taxonomy. We have provided a detailed description of available taxonomies of DTN routing in Section 3.

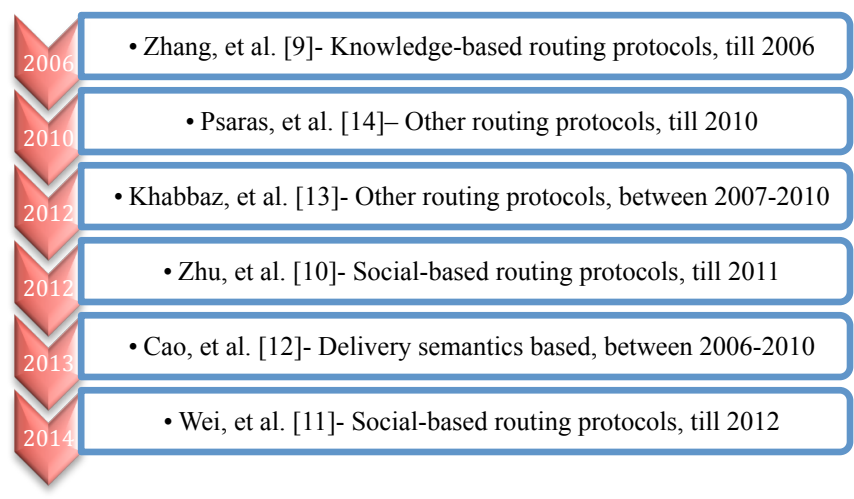

Fig. 2. Timeline of Existing Surveys on DTN Routing

Another categorization possible in DTN routing protocols is based on the single or multiple copies of the messages to be delivered. While message replication improves delivery ratio, it incurs higher delivery cost (delivery ratio and cost metrics are defined in Section 2).

In this paper, we have introduced a new taxonomy to investigate the relation between message replication and packet delivery semantics (unicast, multicast, and anycast) of social-based as well as pure opportunistic forwarding strategies in DTN. Since, the Internet applications are largely moving towards a peer-to-peer information-centric networking (ICN) approach, we investigate how DTN could fit such scenario. ICN evolved from the Content Centric Networking (CCN) paradigm and it aims to route data based on content names instead of IP addresses. ICN shares many similarities with DTN, such as, requirements of in-node storage, late binding, and flexibility in routing (focuses on data delivery instead of end-to-end communication) [15]. However, DTN was developed as a host-to-host communication model which does not directly fit into the content-centric communication model of ICN. Although there exist data-centric opportunistic communication architecture, like Haggle [16], it does not route data using content names as in ICN.

Information Centric DTN (ICDTN) [15] is an effort to integrate ICN with DTN, for a mobile human network. 
Monticelli, et al. [17] have proposed a delay tolerant ICN for controlled information dissemination in emergency and disaster scenes. Anastasiades, et al. [18] have proposed one-hop content discovery in opportunistic CCN, for distributed wireless broadcast environment. They further extended their work [19] for short-term opportunistic network contacts and proposed content centric data transmission using extended caching. Yu, et al. [20] have proposed DT-ICAN, an ad-hoc ICN architecture, which allows the network to adapt to the DTN mode, in the scenario of disruption in connectivity. Batista, et al. [21] have proposed ICON, a new information centric architecture for opportunistic networks. DMND [22] is a NDN based system for collecting data from moving vehicles in presence of intermittent connectivity. Vehicular NDN (V-NDN) [23] is another such NDN framework which allows vehicles to communicate in both infrastructure-based and ad hoc environments.A new adaptation of ICN for mobile environment is called Mobile ICN (M-ICN). The M-ICN network faces frequent partitioning and content requests suffer large delays to reach content producers. As, in M-ICN environment, there may not be a pre-existing path between a pair of nodes, an intermediate node (relay) has to store and carry content requests till it finds an opportunity to forward it. When more than one suitable relay is found, intelligent decision has to be made to choose the relay which can fetch the requested content faster. Disaster zones [24] are ideal application domain of Mobile ICN.

Internet of Things (IoT) is another emerging paradigm which interconnects sensor-augmented smart physical objects. Allowing delay tolerant communication in IoT will enable smart objects to communicate in presence of disruption in connectivity. E.g., in case of remote healthcare IoT applications with limited connectivity, health sensors recording vital signs of patients may need to store/carry the sensed data until it finds an opportunity to forward. Domingo, et al. [25] have proposed an IoT of underwater objects where DTN routing techniques are required to address the intermittent network connectivity. Another example is Opportunistic IoT [26], where connecting devices share and exchange data over an infrastructure-less network (e.g. Bluetooth or Wi-fi) and through opportunistic contacts among humans. Al-Turjman, et al. [27], have introduced a combined RFID and sensor platform which uses delay tolerance in case of intermittent connections.Existing surveys on DTN technology have not looked into the possibility of incorporating delay tolerance features into the aforementioned emerging application areas, which we have covered in our paper. In this paper, we have introduced a new taxonomy to investigate the relation between message replication and packet delivery semantics (unicast, multicast, and anycast) of social-based as well as pure opportunistic forwarding strategies in DTN. Also categorization of routing/ data dissemination protocols based on how relay nodes are selected can be effectively utilized while developing any applications which need to handle disruptions in connectivity.

Table. 1. List of abbrevations

\begin{tabular}{|l|l|l|l|}
\hline DTN & Delay Tolerant Network & ICDTN & Information Centric DTN \\
\hline M-ICN & Mobile Information Centric Network & VNDN & Vehicular NDN \\
\hline NDN & Named Data Network & M-ICN & Mobile Information Centric Network \\
\hline IoT & Internet of Things & RFID & Radio Frequency Identification \\
\hline MoSoN & Mobile Social Networks & GPS & Global Positioning System \\
\hline MANET & Mobile Ad hoc Networks & VANET & Vehicular Ad-hoc Network \\
\hline PSN & Pocket Switched Networks & TTL & Time To Live \\
\hline ICN & Information-centric networking & QoS & Quality of Service \\
\hline CCN & Content Centric Networking & DoS & Denial of Services \\
\hline
\end{tabular}

\section{Performance metrics for DTN routing strategies}

In this section, we shall discuss the available performance metrics in the associated literature of DTN routing and data dissemination. Because of the intermittent connectivity and dynamically changing topology of DTNs, developing an efficient routing protocol should satisfy the performance requirements such as high delivery rate (ratio), low delivery overhead (latency and cost), etc. Similarly, many of the DTN application scenarios use energy-constrained devices, so energy is also an important aspect to consider for evaluating the routing performance. There are some performance requirements specific to the communication paradigm (e.g. multicast). So, we studied the performance evaluation of existing DTN routing protocols and summarized major performance metrics in the existing literature below, for the benefit of future researchers.

- Delivery Ratio: It is the ratio of total number of messages delivered w.r.to the total number of messages sent. This metric determines correctness and effectiveness of the forwarding schemes. Achieving acceptable delivery ratio encompasses intelligent relay node selection or having enough copies of the message in the network, so that at least one of them reaches the destination.

- Delivery latency: It measures the average delay between the time a message is generated at a source and the time it reaches the destination. It captures how efficiently the path is chosen for forwarding. Delivery delay can be low if the relay node is chosen intelligently (moves faster and sooner to the destination) or if there are several copies of a message in the network. 
- Delivery cost: It is measured in terms of the total number of copies of a message generated for routing. Obviously, delivery cost in single copy schemes is less as compared to the multi-copy schemes. However, cost can be reduced in multi-copy schemes by efficiently choosing relay nodes. Delivery cost depicts the overhead of the routing scheme.

- Energy efficiency: It measures average number of messages delivered at the expense of unit energy. Since, power is a limited resource in handheld mobile devices, this metric is very useful for performance measurement. Energy consumption depends upon the quantity of information exchanged and the number of times the message has to be relayed according to the adopted routing protocol.

- Data efficiency: It is the ratio of the number of unique multicast packets successfully delivered (to the receivers) to the total data traffic generated in the network.

- Overall efficiency: It is the ratio of the number of unique multicast packets successfully delivered (to the receivers), to the total traffic generated (both data and control packets) in the network.

- Coverage: It is the percentage of relevant destinations holding a copy of the message when the time to live (TTL) value of the message expires.

\section{Existing Routing and Data Dissemination Taxonomies}

In this section, we study routing and data dissemination taxonomies that have been so far adopted in literature. We have already introduced in Section 1 about the different available surveys on DTN routing protocols. Here we shall cover the major taxonomies adopted by them to classify the existing DTN routing protocols.

Based on the availability of knowledge about the network topology DTN routing schemes have been classified by Zhang, et al. [9] into deterministic and stochastic groups. Below we summarize them.

Deterministic: In this class, the schemes assume that information about the network topology is known apriori. The sub-categories in this class include tree-based, space and time based, and modification-based schemes. In tree based techniques, all nodes have global knowledge about the movement of nodes and contact times. The source constructs a tree, from which, a path with minimum number of hops to reach the destination is selected. In space and time-based schemes, nodes acquire knowledge by exchanging information amongst them and choose the route from this partial knowledge. The final modified shortest path based technique [28] enhances the space and time-based schemes by storing the past path information within the message.

Stochastic: In this class, the schemes assume that no prior information about the node movement or network topology is available and thus, path selections are based simply on heuristics, e.g., forwarding to neighbor nodes (epidemic routing [29]), or based on the mobility pattern of nodes, or link forwarding probability based [8].

Khabbaz, et al. [13] have classified existing forwarding schemes in DTN into opportunistic, probabilistic, vector-based, load-balancing based, encounter-based, resource-allocation based, network coding based, spray-and-wait, and delegation forwarding. They have also surveyed schemes to introduce cooperation and to reduce selfishness among DTN nodes. The authors listed various requirements a good routing protocol should meet to overcome the limitations of DTN. The time span of the papers covered in this work is from mid 2007 to June 2010.

Zhu, et al. [10] have presented a survey on social-based routing protocols. The positive characteristics of social relationships include the social metrics like betweenness centrality, closeness centrality, degree centrality, community, etc. The negative characteristics of social relationships are characterized by the selfishness of the DTN nodes. The schemes using the negative characteristics for routing data are classified into reputation-based, tit-for-tat based, and credit-based categories.

Wei, et al. [11] have extensively studied the taxonomy of social aware routing protocols. They have analyzed the sources of social relations such as, self-reported and detected social networks. In self reported social networks, social information is collected either from questionnaire or from online social networks such as Facebook, Twitter, etc. Social information is collected from detected social networks using mobility traces, call records, and location based services (using GPS or Wifi). The characteristics of social relations, such as social metrics and community structure, are also discussed. The social metrics include degree centrality, closeness centrality, betweenness centrality, bridging centrality, page rank, clustering coefficient, similarity, and selfishness. The community structure, detected with the help of community detection algorithms, is used for identifying the social relations. They have also discussed the optimization strategies for improving the performance of DTN routing protocols.

Although both the above mentioned surveys discussed the social-based routing protocols, in [10], only the characteristics of social relations (both positive and negative) were considered, whereas in [11], sources of social relations together with social characteristics were considered. But none of them included non social-based routing protocols as well as social-aware data dissemination strategies. In our proposed taxonomy, social and non-social (pure opportunistic) routing protocols are discussed based on the packet delivery semantics, such as unicast, multicast, and anycast together with selection of single or multiple copies of messages at source.

Psaras, et al. [14] have discussed about the service targets of DTN protocols such as, high delivery ratio and low 
delivery delay, as well as, system constraints such as, energy and storage. They have classified the routing protocols into flooding/epidemic (also called, replication based) such as, epidemic routing [29], probabilistic and history based [8][30], knowledge-based [28], and social behaviour based [31][32] categories.

Cao, et. al. [12] have classified DTN routing protocols as naive replication based, utility forwarding based, and a hybrid of the two. This classification of routing protocols is based on their design characteristics. The top-level category of such taxonomy is unicast, multicast, and anycast. The naive replication strategy relies on multiple copies of messages for a guaranteed delivery. The utility based techniques use utility metric for relay selection and use a single copy of the message for the delivery.

Data dissemination over DTN is a major application covered in literature which focuses on delivering data from source to destination and is more like a routing approach for the application layer. Data dissemination is done using various approaches, like Pub/Sub achieved using a Pub/Sub based communication paradigm. A survey on data dissemination in VANET is proposed by Wai Chen, et.al. [33], which addresses the geocast/broadcast, multicast and unicast based approaches. A recent survey on routing in vehicular DTN is proposed by Benamar, et al. [34].

In this section, we have discussed the existing survey papers on DTN routing protocols. Most of the survey papers discussed about the DTN architecture, routing approaches [9] [10], design related issues [11], recent developments [12], challenges [13], and application scenarios [33] [34], etc. But none of them focused on the possibility of applying the DTN approaches to emerging areas like IoT, content-centric networking and similar smart application domains. Since DTN's have been studied by researchers for more than a decade, incorporating delay tolerance to emerging applications should be the further focus of research, instead of developing stand-alone DTN routing protocols. We have already investigated in this survey, about some of recent developments in futuristic applications of DTN. We also discussed about data dissemination literature in this paper, which we feel as the most appropriate for developing such delay tolerant futuristic applications and which has not been addressed in any of the existing surveys.

\section{A Novel Taxonomy of Routing and Data Dissemination in DTNs}

Existing survey papers on DTN routing approaches have focused on different aspects, such as, social-based and pure opportunistic forwarding techniques. In this paper, we focus on a comprehensive study of DTN routing and data dissemination approaches in a layer-wise manner.

In Fig. 3, we have segmented DTN routing and data dissemination issues, based on the operations/functions carried out. The lowest layer determines the number of replicas to be produced for a DTN message to maximize the delivery opportunity. While some researchers opt for a single-copy of the source message and depend on higher layers for guaranteed delivery, others prefer to inject multiple copies of the same message (routed through different relay nodes) with a hope that at least one of them will find the destination. One extreme of multi-copy routing is Epidemic flooding [29] where at every intermediate relay node, the message is forwarded to all neighbor nodes but the source.

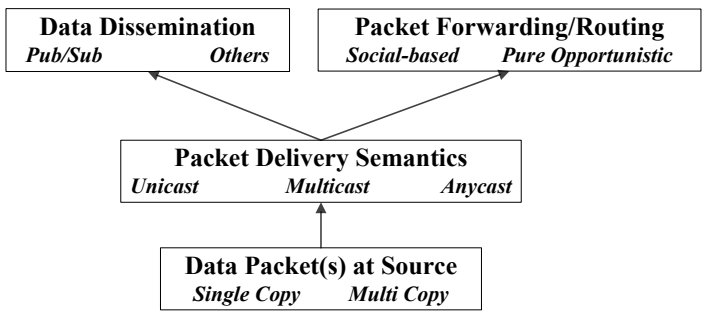

Fig. 3. Layered Structure of DTN Routing Techniques

After being injected into the network from the source, packets are relayed by other nodes following unicast, multicast, or anycast communication strategies depending on the number of receivers. However, such communication strategies are not strictly related to the number of copies the source injects, e.g., multi-copy source messages may have single [29][35] or multiple [36] message recipients. Similarly, a single copy message is compatible with any of the aforementioned communication strategies.

The top layer determines the strategies to be adopted for forwarding data packets using either social metrics (friendship, community structure, centrality measures, etc.) or pure opportunistic contacts. We have also incorporated the data dissemination in DTN alongside the packet forwarding techniques as most of the data dissemination protocols in DTN adopt social-based and pure opportunistic techniques for disseminating data. Data dissemination in DTN can be achieved by using a Publish/Subscribe (Pub/Sub) Overlay. Pub/Sub decouples the communication entities and supports asynchronous delay-tolerant communication. 


\section{DTN Routing Strategies}

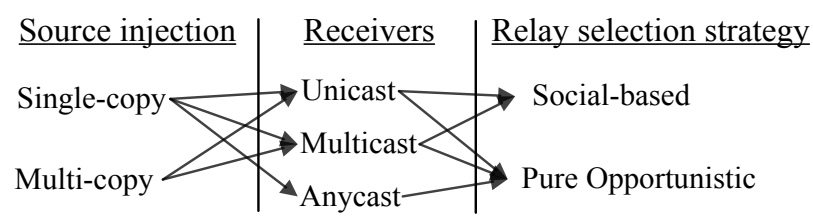

Fig. 4. Classification of Routing Strategies in DTN

The diagram shown in Figure 4 captures different combinations of the DTN routing parameters (number of source data packets, forwarding decisions, and delivery semantics) introduced in Fig. 3 for classifying existing DTN routing protocols. Single copy source message may be intended for any number of recipients and therefore, may adopt unicast, for a single recipient, and multicast or anycast for group communication. Multi-copy source messages may be intended for a single user or for a group and unicast or multicast is used, respectively to achieve the delivery objective. While the joint usage of such techniques is possible, and might also be useful in given scenarios (e.g., when employing source coding techniques), we did not find any multi-copy message delivery using anycast semantics.

In summary, we provide a novel taxonomy for classifying existing DTN routing and data dissemination protocols. Due to the inherent nature of DTNs to support communication in the presence of disruptions in connectivity, there are plenty of opportunities for delay tolerance support in several emerging network-based application domains, such as IoT, ICN, NDN, etc. Since, the research in DTN is almost matured; our motivation is to point out to the future researchers the need of delay-tolerance support in emerging networking applications. The advantage of our proposed taxonomy lies in the fact, that it will help the future researchers to have a deeper understanding of the existing literature from a futuristic perspective.

\subsection{Taxonomy of Routing in DTNs}

In this paper we classify existing DTN routing protocols based on the approach adopted for selecting intermediate relay nodes for carrying the message towards the destination. In fact, DTN literature has so far adopted either social-based or pure opportunistic interaction for relay selection. Social-based opportunistic forwarding requires finding a relay node with high betweenness centrality, high popularity rank, etc. Such values are shaped by the social characteristics of a node: interaction history and node mobility patterns are the key factors. On the other hand, this same information can be put to good use in pure opportunistic forwarding to find a relay node which has a higher chance of routing a given message towards its intended recipient. While performing this, the probability of delivering a message is predicted for all the nodes.Based on that, only the nodes that have a higher delivery probability will receive messages, thus reducing the total number of copies required to deliver (and the delay incurred by) a given message. Another approach can be flooding based schemes, where the overhead of finding better relays is replaced by message overhead in terms of more copies in the network. 


\section{Social Based Routing}

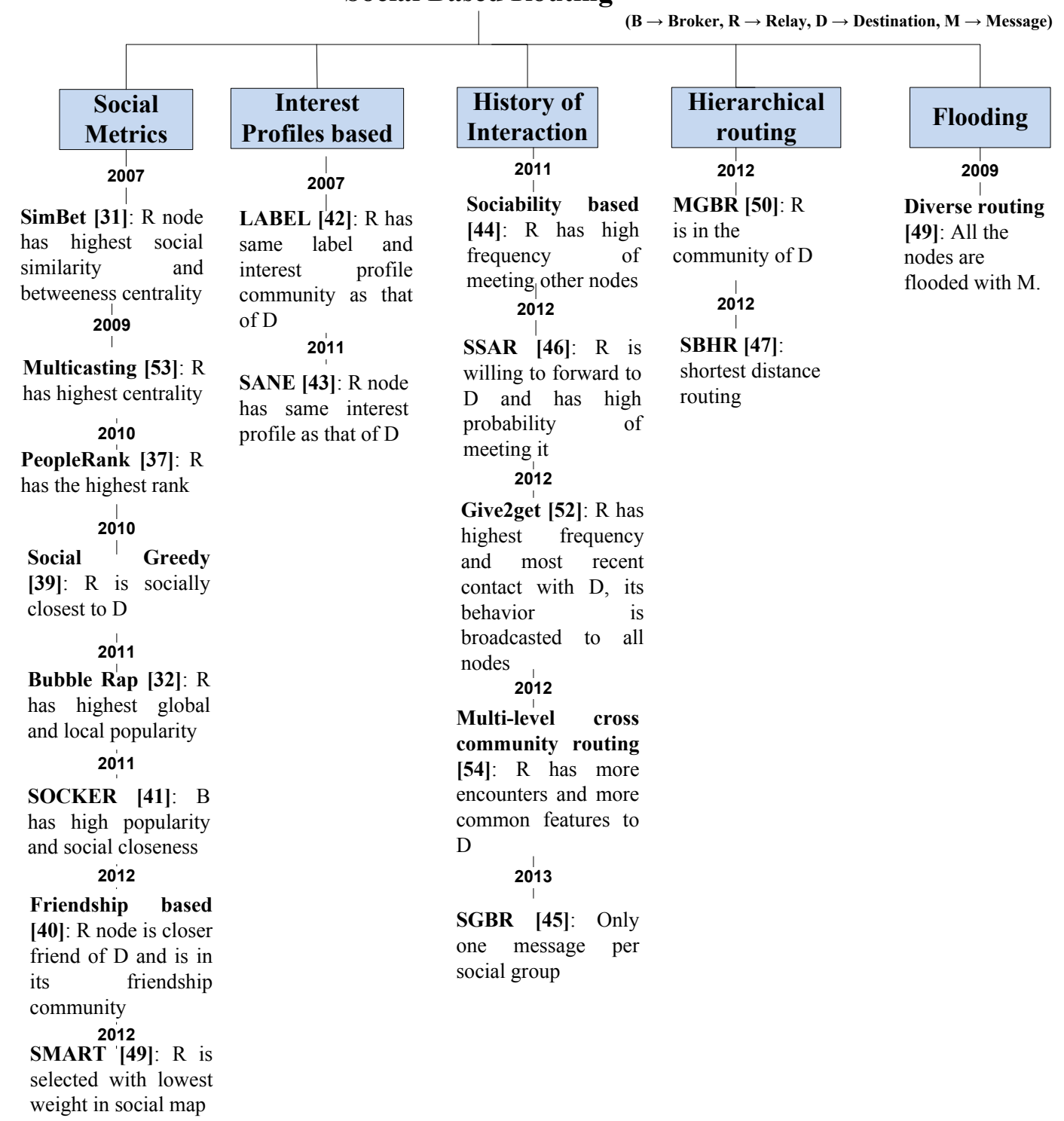

Fig. 5. Classification of Social-based Routing in DTNs

\section{Taxonomy of Social-based Routing in DTN}

Based on the criteria of relay selection for message forwarding, we distinctly classify (Fig. 5) social-based DTN routing protocols in the following categories.

- Social metric: They utilize a social metric to select the relay node.

- Interest profile: Node interest profiles are put to good use to select a relay. Nodes with similar interest profiles as that of the destination of the message are chosen as relays.

- History of Interaction: Past mobility information is exploited for node selection. As an instance, a node having the most recent and the highest number of encounters with the destination is selected as a relay.

- Hierarchical: The routing of the message is done from higher level communities to the lower level communities, if no appropriate relay is found in the higher level communities.

- Community Flooding: The whole community of the source node is flooded with messages and then through intercommunity movements of nodes, the message reaches its destination. 


\section{Taxonomy of Pure Opportunistic Routing in DTN}

Pure Opportunistic routing has been developed in the literature with a number of different techniques devised for taking routing decisions. We have classified existing pure opportunistic DTN routing protocols in the following categories (Fig. $6)$.

- Dedicated nodes based: includes those routing schemes where special dedicated nodes are assigned the task of collecting and delivering messages to the source and destination nodes. The regular nodes, in these schemes do not participate in message forwarding.

- Routing criteria/metric based: schemes where relay selection is based on certain routing metrics, such as, shortest distance to the destination, average or expected delay towards the destination, shortest path to the destination, etc.

- Hierarchical: these protocols consider the network as a hierarchical structure, with source as the root node and the destination(s) as the leaf nodes.

- Prediction based: includes those schemes where something about the set of nodes, from which the relay is to be selected, is predicted. Be it the delivery probability, the future encounter with the destination or time of encounter with the destination.

- Clustering based: includes protocols which use network partitions, based on, e.g. the node density in a region, etc. The routing is done between these clusters.

- Probabilistic: nodes calculate some probability values to make the forwarding decision. It may be the probability of the message reaching the destination or the delivery probability of a node, etc.

- History of encounters: nodes record their encounters with other nodes and use this information later in relay selection. In some schemes, the relay can be the node with highest frequency of encounters with the destination, while in others; it can be the one having highest frequency of visiting a location of the destination.

- Coding based: such strategy entails coding a message, which can be fragmented and later reconstructed, to form the original message. These codes can be independently routed towards the destination.

- Flooding: the simplest idea for routing, but it has evolved to impose certain restrictions on the number of copies. The replication probability can be dependent on the number of copies present in the network or the expected delivery delay of the message.

\subsection{Taxonomy of Data Dissemination in DTNs}

Data dissemination in DTN addresses the issues of many-to-many, any-to-many, and one-to-any communication for sharing and distribution of data. This can be done through some social community detection methods, or by leveraging history of encounters between nodes, as well as prediction methods. Therefore, data dissemination in DTNs, like routing strategies, can also be classified into social-based (Fig. 7) and pure opportunistic (Fig. 8) classes.

Social-based data dissemination schemes can be classified in two families: those that use the Pub/Sub paradigm and those that do not. A publisher is a node which has some data to give away to the nodes which have subscribed to it, called subscribers. The publisher may not be aware of the subscribers and their locations. The peculiarity of Pub/Sub based schemes are, hence, that they allow one-to-many communication, whereas non-Pub/Sub schemes offer many-to-many communication. Both Pub/Sub and non-Pub/Sub based approaches, however, typically rely on either social metrics or on utility functions to disseminate data. Social metric based algorithms use social metrics like centrality to disseminate data. Utility based approaches, on the other hand, compute utility of the data items as a function of their demand and find the nodes which optimize this utility function.

Pure opportunistic data dissemination can be either push-based or pull-based. They can also be called as reactive and proactive approaches, respectively. In reactive approaches, the source node(s) push the message/data towards the intended recipients and in proactive approaches, the subscribers pull the data/message from the nodes which carry that data. We identified four different reactive approaches for data dissemination. Common nodes based data dissemination considers the less prominent nodes as carriers for the data towards its recipients. Another approach ranks the data by its popularity. Coding based schemes use various coding techniques, so that, if only a few of the code blocks reach the subscribers, they can retrieve/reconstruct the whole message from those few blocks. In the last approach, the publisher uses prediction of the subscriber's location (from mobility pattern) and limited flooding to disseminate data towards the subscribers. 


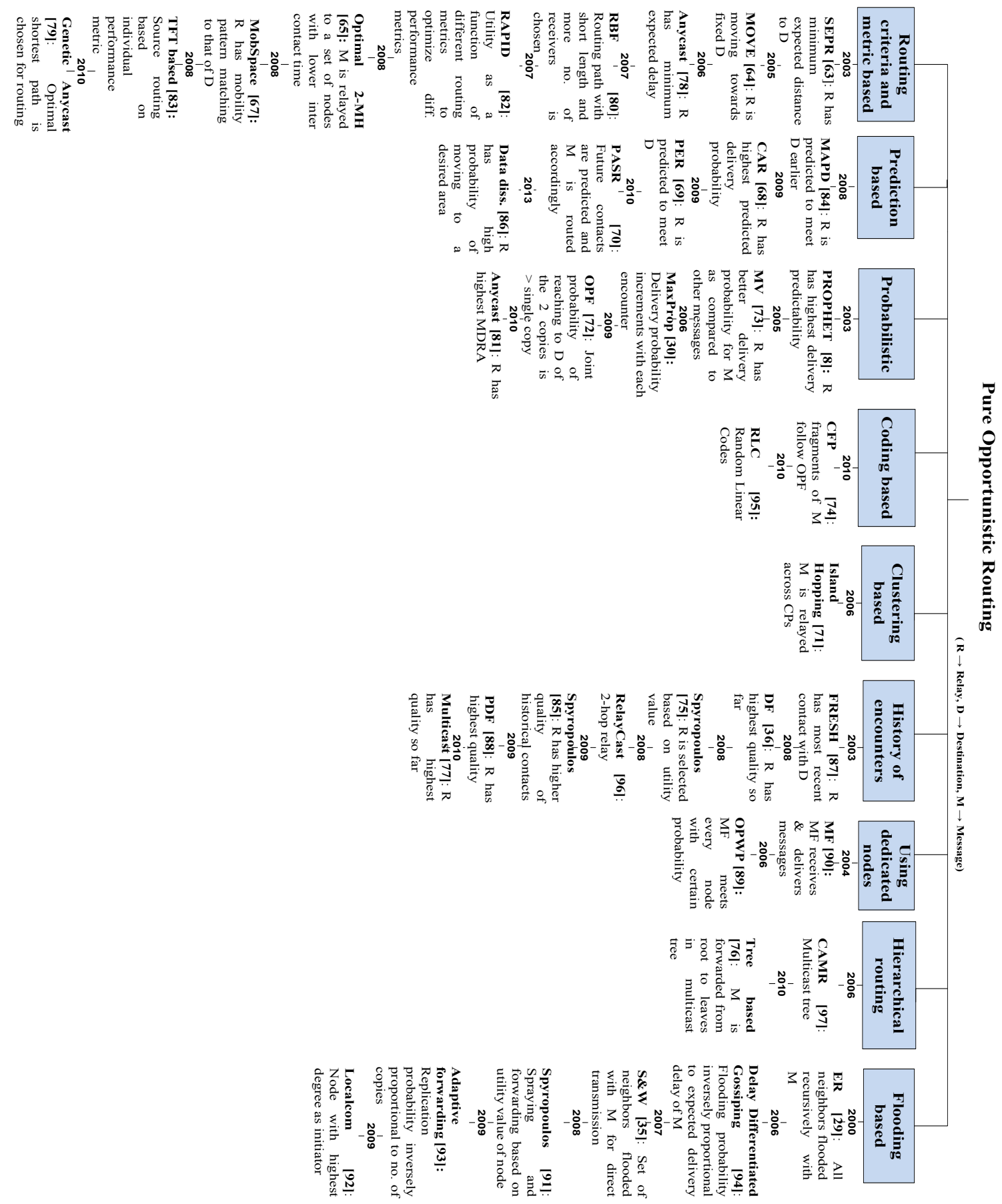




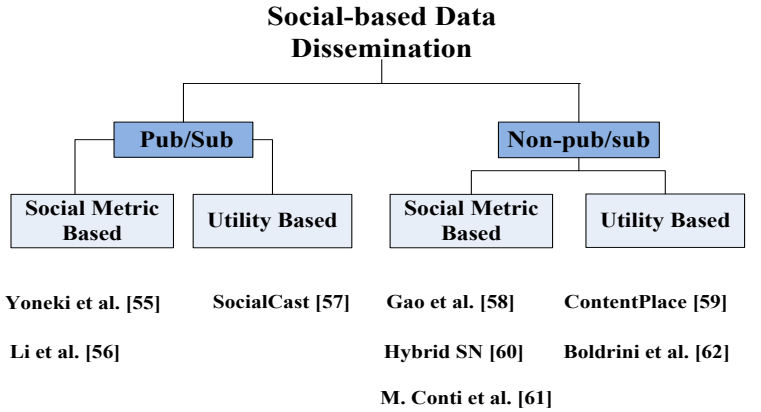

Fig.7 Classification of Social-based Data Dissemination in DTN

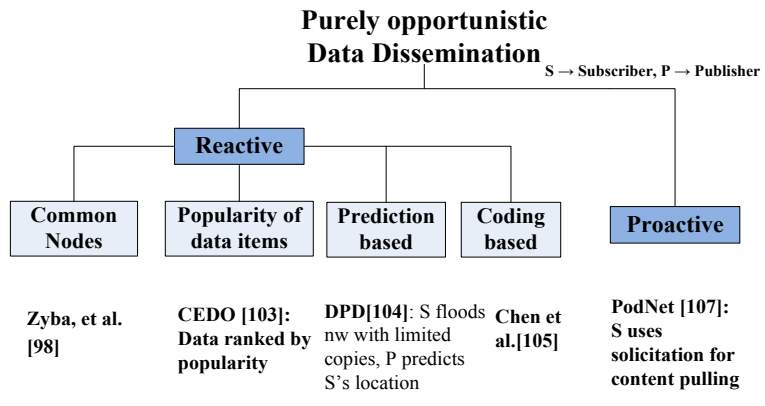

Fig. 8. Classification of Pure Opportunistic Data Dissemination in DTNs.

\section{Social-based Routing Protocols in DTN}

In this section, we shall discuss the single-copy, unicast, social; multi-copy, unicast, social; and multi-copy, multicast, social protocols in Section 5.1, 5.2, and 5.3, respectively.

\subsection{Single Copy, Unicast, Social}

\section{Social Metrics}

In Simbet [31] routing, every node chooses a relay node among its neighbors by locally computing betweenness centrality and social similarity metrics. Simbet chooses the node with the maximum value of Simbet Utility, a weighted combination of betweenness centrality and social similarity, as the relay node.

PeopleRank [37] is yet another effort towards identifying the most popular node in the social graph and it is inspired by the Google's PageRank algorithm [38]. The popularity of a node is termed as its Rank and nodes with higher Ranks are chosen as relays with the basic assumption that they are more likely to meet other nodes. Experiments on real mobility traces prove the efficiency of the proposed scheme.

Bubble Rap [32] is another social metric based DTN routing protocol, which uses community and centrality measures. Every node belongs to one or more communities and their popularity inside each community is measured by either degree or betweenness centrality of the node. Global popularity is measured by calculating centrality of a node considering the entire social graph as a single community. Messages are forwarded to nodes with higher global popularity until they reach the local community of the destination node, in which case, a node having higher popularity in that local community is chosen as a relay.

Social Greedy [39] uses a greedy approach for message forwarding. It calculates social distance (or, closeness) between nodes using Small-world network model. The message is forwarded to a node if it is socially closer to the destination node than the one currently carrying the message.

Friendship based routing [40] considers friendship as a social metric for choosing relay nodes in DTN routing. The degree of closeness among friends is determined by several behavioral features, such as, frequency and regularity of contacts. It is assumed that close friends see each other more often and can prove to be better relays for message routing. Nodes having friendship values higher than a threshold are grouped into a friendship community. Messages are forwarded to a node iff it is in the destination node's friendship community and it is a close friend of the destination node than the node currently carrying the message.

SOCKER [41] is a dynamic community creation mechanism based on social broker selection strategies, in which dynamically selecting a broker during each opportunistic encounter and selected broker disseminates community creation requests to the encountered users for match making. In case of broker/relay selection mechanism in opportunistic networks, two metrics, social closeness and user popularity, are used. Whenever a new node joins the network, then the broker selection process is initiated.

\section{Interest Profile Based}

In LABEL [42], each user is assigned a label which identifies his or her interest or affiliation and users with the same label are grouped into a community. Relay selection is based on the idea that the users of same community tend to meet each other more often than users in different communities. So, messages are either forwarded to the destination node or to any relay node belonging to the community (having same label) of the destination node. The authors carried out experiments on human mobility traces and established the improvement in delivery ratio and cost over existing schemes. 
In SANE [43], the interests of people are used to make the forwarding decision, with an assumption that people with similar interests meet frequently. Similarity of interest is modelled as cosine similarity. The interest profile of the destination is stored in the message as the message reference profile. Each message $M$ has a header that contains this message reference profile, together with the number of replicas, $n$, of the message that a node is allowed to forward and the TTL. As there is no need to store any other information apart from interest profile, it is a stateless routing protocol. Relays are selected based on similarity between its interest profile and the message reference profile. If this similarity is greater than a fixed threshold, the message is forwarded and the number of replicas is decreased by half $(n / 2)$. As compared to Epidemic routing [29] and Spray and Wait [35] schemes, this protocol achieves better coverage and lower delay.

\section{History of Interaction}

Another social metric, introduced in [44], for choosing relay nodes, is called sociability which identifies highly socially active nodes based on their number of social interactions with other nodes. A set of nodes with a high degree of sociability is chosen as a restricted set of relays which have higher chances of meeting the destination nodes. Sociability of a node is calculated either considering interactions with its immediate neighbors (first hop-based sociability) or by the interactions of its neighbors with their neighbors and so on, in an iterative way (K-th hop-based sociability). K-th hop-based sociability stresses the importance of having highly sociable neighbors in forwarding bundles.

In SGBR [45], nodes divide themselves into groups. Two nodes are in the same group if they frequently contact each other; there is no need to know the network topology a priori. The relay is selected based on high contact frequency. The routing is done such that, there are less number of packets so as to reduce the cost. It is done by checking before transferring the packets that whether the receiving node already had the packet beforehand and by keeping only one message in a group. Each node in a group can forward packets to different groups. The messages are dropped from the network when the TTL becomes zero. With the help of experimental results on the real data trace it is proved that SGBR produces a greater delivery and less network overhead ratio than PROPHET [8] and Epidemic Routing [29].

Li, et al. [46] proposed SSAR (Socially Selfishness Aware Routing), which utilizes user's selfish behavior. To select a forwarding node for a message, both, node's willingness to forward to this destination and the probability of its contact opportunity with this destination, are taken into account. When two nodes discover each other as neighbors, they deliver the messages destined for each other; the messages are delivered from highest priority to lowest priority. When a node A has extra messages (not destined for its neighbor B), A will send the summary of all the messages to node B. Node B then calculates the new priority of the messages according to willingness to forward and the delivery probability for each of the message. It then sends all of this information to node A. Node A decides which messages to forward to node B with the help of the multiple knapsack problem solution. SSAR works in two modes, forwarding and replication. In forwarding mode, when node A forwards the message to node B, node A deletes the message from its buffer. In replication mode, there can be multiple copies of the messages present in the system. Replication mode gives a higher delivery rate, but uses more resources than forwarding mode. The experimental results carried on real data trace for forwarding mode shows that the algorithm works better than PROPHET [8] and SimBet [31], in terms of delivery ratio.

\section{Hierarchical Routing}

A social-based hierarchical routing scheme is proposed in [47] which use hierarchical community structure. The lower of the two-layer hierarchy contains individual social communities comprising of nodes and links representing social relations between a pair of nodes. The higher layer records relations among multiple communities, overlapping or non-overlapping. While overlapping communities are merged, partially overlapping communities are represented as graph nodes linked by an edge whose weight is determined by the number of common member nodes. No link is present between nodes representing non-overlapping communities. Message forwarding is carried out following the shortest-path among the communities or the nodes of a community in inter-community or in intra-community environment, respectively.

\subsection{Multi Copy, Unicast, Social}

\section{Social Metrics}

In SMART [48], a routing scheme is proposed which requires construction of a social map with 1-hop or 2-hop neighbors. For each node listed in a node's top friends list, ranks are assigned which represents meeting frequencies of a node with others; the highest met friend is assigned a rank 1 and so on. By exchanging this information node creates a social map. In the map, the weight of the social link combines all the paths between two nodes. Dijkstra algorithm is used to find the shortest path between two nodes in forwarding. The algorithm always forward packet to the node with the lowest weight. After receiving the packet, if the storage is full at the receiver's side, then the receiving node drops the packet which is long lived. Experiments on real data trace prove that, SMART has a higher hit rate and lower average delay than direct 
transmission, SimBet [31] and Prophet [8] algorithms.

\section{Flooding Based}

In Diverse routing [49], the average inter-encounter interval is used as a social metric to cluster the network. A node is said to be a social neighbor of a node if the inter-encounter interval between them is above a threshold. Messages are spread to all the social neighbors of the node recursively until the message reaches the destination. Experiments on reallife traces show improvements in worst-case latency.

\section{Hierarchical Routing}

A multi-level group based routing [50] protocol is proposed in which the relay is selected based on the social group information, which is generated from node's encounter history. A content graph is created, in which vertex denotes the nodes and edges denotes the history of their meeting. Multi level social groups are formed with the help of number of past encounters among the nodes, i.e., if the number of contacts between two nodes is more than a particular threshold, then they will be having an edge between them. After the group is formed, each connected component is treated as a new group. In routing with single-level social groups, the node $i$ pass the message to node $j$ only if the node $j$ is in the community of the destination node of the message M. In Routing with multi-level social groups, the social groups are formed based on decreasing threshold values and the relays are searched from group one to group $\mathrm{n}$. This concept is named as mGroup and experimental results on NUS contact trace [51] data show that the method gives a better delivery performance than the single level approach, epidemic routing [29] and greedy algorithms.

\section{History of Interaction}

Give2get (G2G) [52] proposes a pair of forwarding protocols for DTN amidst the extreme situation, where every node is considered to act selfishly. One of the protocols is G2G epidemic forwarding, which relies upon cryptography and every node has to forward two copies of the message to their first encountered nodes. The selfishness of the nodes are mitigated by broadcasting about the behavior of such nodes. The other protocol is G2G delegation forwarding in selfish environment. The authors in G2G suggest that the existing forwarding quality metric can be hampered in presence of selfish nodes. So, they proposed that relay node should be selected based on both number of encounters and recent contacts with the destination. Through simulation with real mobility traces they have proved that G2G protocols follow Nash equilibrium, i.e., nodes have no incentive to deviate (or to show selfish behavior) from the prescribed protocol.

\subsection{Multi Copy, Multicast, Social}

\section{Social Metrics}

Multicasting on both single and multiple data item based on centrality is introduced in [53]. In case of delivery of a single data item, cumulative contact probability is used to model centrality. A node with highest centrality value is selected as a relay from a set of neighbors. In case of delivery of multiple data items, the authors have introduced another centrality metric which relates to the probability of delivering a message to a node. A node having higher value of this centrality is selected as a relay. A node calculates delivery probability for every message and the data item having higher such probability is forwarded to the relay node.

\section{History of Interaction}

Li, et al. [54], have introduced a group-based routing protocol for wireless DTN. Nodes are connected to form a contact graph, if they have ever encountered each other. Higher number of encounters results in stronger edge weights in the contact graph. A separate graph called, social feature graph is also formed by connecting nodes having identical values of specific social features, such as nationality, languages, etc. Edge weights in the social feature graph equal to the number of identical social features. These two types of graphs are merged to form a hybrid social graph called, multi-level cross community social graph, which is used for data forwarding. Data forwarding in the hybrid graph is achieved on the assumption that people having more common social features tend to interact/contact each other more frequently.

\section{Social-based Data Dissemination in DTN}

Social-based data dissemination schemes have been classified into two types - Pub/Sub based and using other techniques (not pub/sub based).

\subsection{Using Pub/Sub Paradigm}

Social Metric Based

Yoneki, et al. [55] have proposed a Pub/Sub based data dissemination method. A community is formed by interconnecting 
nodes that meet frequently with each other. In each community, a node with the highest closeness centrality is selected as a broker. Brokers form an overlay for cross-community message exchange using gossip style communication. Experiments carried out using real human connectivity traces show highly reliable data delivery with low latency.

Li, et al. [56] have also proposed a Pub/Sub scheme, called MOPS, for data dissemination over DTN. Nodes form closeness-based, completely connected community graphs, where closeness is determined by average inter-contact period between two nodes. Nodes exchange interests and events in a peer-to-peer manner within a community. For intercommunity Pub/Sub support, nodes bridging neighboring communities are used as brokers which help to fetch as well as disseminate events and interests, respectively. Simulation over real and synthetic mobility traces proves that MOPS outperforms pure push and direct pull strategies for data dissemination in DTN-type network. All the communications were based on gossip style data exchange for each encounter.

\section{Utility Based}

SocialCast [57] introduces a routing scheme for Pub/Sub based data dissemination using social relations between nodes. Since, socially close individuals tend to stay together, they use co-location property to disseminate data from publishers to subscribers. Initially, subscribers disseminate the events they are interested in by broadcasting to their immediate neighbors, which may act as carrier nodes or brokers. Publisher nodes create source message and carries multiple copies of it. When a publisher encounters a carrier, the latter performs a matching of the stored subscription interests and forwards to the interested subscribers. Messages are forwarded from a broker to another broker sporting higher utility value.

\subsection{Using Other Techniques}

\section{Social Metric Based}

A social-metric based data dissemination scheme has been proposed by Gao, et al, [58]. They used a modified version of the well-known centrality metric for relay selection for data dissemination. Their centrality is a combination of social contact patterns of nodes and probabilistic measure of their interests in specific data items. Data forwarding is done to optimize cost effectiveness, which guarantees timely delivery of data items to subscribers by choosing the optimal number of relays. Experimental results show that the algorithm works better than ContentPlace [59], flooding scheme and randomflooding based algorithm in terms of the number of interested nodes reached and cost effectiveness.

The authors of Hybrid SN [60] have suggested that treating online and physical communities as complementary entities for data dissemination over DTN can reduce delivery latency while increasing the coverage as compared to individual schemes. Contact duration and contact frequency metrics are used to characterize social relations between two nodes. Two types of brokers, for offline and online communities, are selected based on their social selfishness and popularity respectively.

Social aware content sharing is proposed by Conti, et.al [61], which exploits the user's social behavior and interest for allowing cooperative downloading among users even without a direct connection. User's information is divided into private and public contexts. Private contexts concerns with the personal information of the user and public context related to the service they have subscribed to. An identity table is created for each user which consists of both private and public contexts. A node exchanges identity table with its neighbors and fetches new contents when moving across communities. In such a cooperative downloading, utility function is used to determine which data item to fetch.

\section{Utility Based}

Boldrini, et al. [1] have introduced a novel utility-based data dissemination system leveraging the user's social context. In a furtherance of their work, ContentPlace [59] was introduced, which uses the social utility based model and store data item on certain nodes, so as to optimize availability of data items to the subscribers. Based on their interaction with each other, nodes come to know about the data items requested by subscribers and those made available by the publishers. A pair of nodes, upon encounter, calculates the utility values of all data items currently cached by both of them to decide upon a set of data items to cache individually, in order to maximize their individual local utility. Subscribers receive a subscribed data item when they interact with a node caching the same. Utility values are calculated using a social assumption that different social communities have interests in different data items. So, while calculating utility value of a data item, subscriber's social strength with all the communities it is related, is considered. Experimental results show that the algorithm performs better in terms of hit rate when compared with the access probability and uniform algorithms.

Boldrini, et al. [62] proposed a utility-based data dissemination method for opportunistic networks. Utility values are used for data caching by nodes using a gossip style communication. Utility is calculated using a local and a social component, where the local component checks the interest of the local user in a data item. The social component, on the other hand, checks the interest of its social community members in the data item and whether they have cached it or encountered it in other nodes. 


\section{Pure Opportunistic Routing Protocols in DTN}

In this section, we shall introduce pure opportunistic routing protocols in DTN on the basis of our taxonomy presented in Fig. 6.

\subsection{Single Copy, Unicast, Pure Opportunistic}

\section{Routing Criteria and Metric Based}

In SEPR [63], expected path length is used as the forwarding metric. Every node calculates its expected path length to the destination using Dijkstra's algorithm. Every message is associated with an effective path length, which is the minimum of the expected path lengths of the nodes the message has passed through. At the source (S), the effective path length of the message is set to the expected path length of S. The message is then forwarded to a node which has an expected path length smaller than the effective path length associated with the message. When a message is forwarded, its effective path length is updated to the relay node's expected path length. Experiments have shown that the method increases the delivery rate and reduces the delivery cost.

The approach proposed in [64] is based on whether the current node and its neighbors are moving towards or away from the fixed destination. Because the destination is considered to be fixed, the angle between the current direction of a node in motion and the direction of the destination is calculated to assess the relative motion and the shortest distance to the destination. Therefore, in this scheme, nodes share their motion vectors with each other to make the forwarding decision. Authors have also proposed MoVe-Look ahead scheme, where the knowledge of the waypoints (where the direction of motion of a node changes) is also exchanges between nodes on encountering each other, for making forwarding decisions. The delivery ratio is high and the delivery cost is low for MoVe algorithm as compared to location based routing and direct forwarding.

The input to the algorithm in [65] is the inter-contact time between the nodes in the network. The relay nodes are selected based on minimal expected delivery time to destination, which is calculated from the inter-contact time between the nodes. As compared to the Direct Forwarding, 2-hop strategy [66], Minimum Expected Delay [28] and Epidemic routing [29], this method yields a better delivery ratio and reduces the delivery delay.

Mobyspace [67] uses high dimensional Euclidean space for selecting a relay node that has matching mobility pattern with the destination. Mobypoint represents the mobility pattern of a node in Mobyspace, which is used for making the match. Each node calculates its Mobypoint from its coordinates in MobySpace, which represents the locations and the frequency of visiting those locations by this particular node. The axis in the MobySpace determines the future nodes that a particular node will encounter. A node selects a relay node to forward, if its Mobypoint is matched to that of the destination.

\section{Prediction Based}

In CAR [68], a node chooses a relay node based on high mobility and past encounter history with the destination. A node calculates the delivery probability for a set of nodes based on context information. Authors have selected the rate of change in connectivity and future host co-location as the context attributes. Context information is calculated by finding the utility values for every context attribute. Kalman-filter based forecasting technique is used for predicting the future values of context attributes and delivery probability. Nodes update and exchange the delivery probability with each other proactively. A node with highest delivery probability is chosen as relay node. Compared with Epidemic routing [29], PROPHET [8], and Spray and Wait [35], delivery ratio is higher in CAR even with small buffers.

In Predict \& Relay [69], the future mobility of a node and the locations it will visit are predicted to calculate the delivery probability of the node. Both space and time are involved in the process of forwarding. A node is selected as a relay if its delivery probability is higher than the node currently having the message.

Guo, et al. [70] have proposed Prediction Assisted Single-copy Routing (PASR), for routing in underwater sensor networks. A greedy algorithm, called Aggressive Chronological Projected Graph (ACPG), has been developed for predicting the future contacts of nodes based on historical information and node mobility. Geographic preference, contact periodicity, inter-contact time distribution, and contact probability are the metrics used for historical information. Kinematic model is used for modelling the mobility. Using ACPG, two single-copy routing protocols have been proposed for routing under different mobility models. Compared to the existing routing protocols, such as Epidemic routing [29] and First Contact [71][28], the proposed methods achieve higher delivery ratio and lower average delay.

\section{Probabilistic}

Liu, et al. [72] have proposed a routing scheme in which every node has the information about the average inter-contact time of all other pairs of nodes. This can be calculated using encounter history (due to the underlying assumption of patterns in node motion) and then sharing it. When a node $\mathrm{i}$ has a message copy $\mathrm{M}$, it will forward the message only when 
the joint probability of meeting the destination of the two copies (from separate nodes) is either greater than or equal to the probability of meeting the destination if not forwarded. If the node i encounters more than one node in the same time slot, it will consider the node which has a higher probability of delivering to the destination. These probabilities can be calculated using a probability metric which is a function of remaining hop counts and the remaining time-to-live. Simulations show that this scheme performs better than delegation forwarding [36] in terms of delivery rate, delivery delay, and cost.

In MV [73], nodes have information about the meetings between various node pairs and their visits to different locations. It uses such information for calculating the delivery probability for messages. On encounter, two nodes exchange messages along with their delivery probabilities. Each node calculates delivery probability of the messages received and keeps only the ones for which it offers the better delivery probability. The authors have also addressed the situations in which there are no relay nodes to select. In such scenarios, autonomous agents like mobile robots are deployed to get information about the state of the network. A controller is designed for controlling the movements of the agents to yield better performance. The MV algorithm achieves a better delivery rate as compared to the FIFO buffering strategy and no buffer strategy.

\section{Coding Based}

In CFP [74], optimal probabilistic forwarding (OPF) is used together with fountain codes. Messages at source are fragmented into frames using fountain codes. These frames can be routed separately and the receiver needs only a few frames to recover the original message. In this way, this scheme saves the network resources. The frames of messages are forwarded by each node based on the optimal stopping rule which is used in Optimal Probabilistic Forwarding (OPF) algorithm [65]. In OPF, a message is forwarded to a relay node if the joint probability of delivery of these two copies is higher than the delivery probability without copying. Simulation results show that the proposed method achieves better delivery rate compared to OPF at the cost of slightly higher delay.

\section{Clustering Based}

Djukic, et al. [71] have proposed a mobility model based on the assumption that the network always has stable concentration points (CPs), where the node density is higher. Thus, at these CPs, the nodes have more communication with each other. The network can, therefore, be represented as a graph with vertices as CPs and edges as the movement of the nodes among CPs. Based on this CP graph, a forwarding algorithm, called Island Hopping, is presented. If the destination of the message is a fixed node, then there can be three ways of forwarding the message (M). The first way is to find the shortest route from source node's CP to destination's CP using the graph, store this information in M and send copies of $\mathrm{M}$ to some of the neighbors. If the nodes carrying $\mathrm{M}$ traverse CPs following the route stored in M, they carry along M, otherwise, they drop it. The second way is to generate copies of M in the current CP. Some of the nodes having a copy of $\mathrm{M}$ will move to other CPs and replicate copies there. If any node having a copy of $\mathrm{M}$ comes back to the previous $\mathrm{CP}$, it means that message has been successfully replicated in the next hop CP. Therefore, the message copies are discarded in the previous CP. The third way is to limit the number of copies in next hop CPs by discarding copies of M. If a second node moves from the previous $\mathrm{CP}$ to the next hop $\mathrm{CP}$, it will produce copies of $\mathrm{M}$ iff there was no such copies pre-existing in the current CP. If the destination is not fixed, the approach is to use the last encounter information of nodes to reach to the destination.

\section{History of Encounters Based}

Spyropoulos, et al. [75] have proposed a single-copy routing protocol for DTN. The method aims to limit the number of copies that has to be transmitted in the existing spray and wait technique. Also, instead of spraying messages to all the neighbors as in [29], each copy of the message is routed separately, i.e., routing decisions are made separately. Relay nodes are selected based on utility value which considers past encounter history of nodes.

\subsection{Single Copy, Multicast, Pure Opportunistic}

\section{Hierarchical Routing}

$\mathrm{Wu}$, et al. [76] have proposed a multicast routing scheme with the concept of the multicast tree. The destination nodes are the leaf nodes in the tree and compare-split rule is used for constructing new branches of the tree. In the compare part, when a node having destination subset encounters another node without any destination subset, both of them exchange the contact probability vectors with each other. Contact probability represents the encounter history of a node with the destination. If the contact probability vector of the second node is higher than the first node, then splitting is performed using ratio-based split. In ratio-based split, the ratio of the active levels of encountered nodes is calculated which is used for splitting the destination set. Active level represents the number of contacts encountered by a node. The advantage of 
compare-split is that the destination can be reached in multiple paths which reduce the cost as well as the delivery delay in multicast.

\section{History of Encounters}

Wang, et al. [77] have presented three multicast algorithms. These are single copy multicast, multi copy multicast, and delegation forwarding multicast. Delegation forwarding multicast is also a multi-copy approach. The quality metric used in this paper is same as that used in delegation forwarding scheme [36]. In single copy multicast scheme, the source of the message will forward its copy only to that encountered node which has higher quality value for all the destinations than the source. As this condition is too strict to implement, a slightly lenient one is to compare only the sum of the quality values of all the destinations for selecting a relay node. In multi copy multicast, the source will forward a copy to a node which has a higher quality value for any one of the many destinations. In the final approach, the existing and well-known delegation forwarding scheme [36] has been extended for multiple destinations. For every destination, the node will forward a copy to an encountered node only if the quality value of the encountered node is greater than all the nodes encountered so far. Out of all these multicast schemes, the delivery delay in the stricter case of the single copy scheme is highest, but at the same time, overhead is also least. Delegation forwarding multicast has a minimum latency among all three schemes and forwards less copies than the multi-copy multicast scheme.

\subsection{Single Copy, Anycast, Pure Opportunistic}

Anycast allows a node to send a message to another node belonging to a group of destination nodes.

\section{Routing Criteria and Metric Based}

The idea presented in [78] is that the route can be dynamically re-decided based on node mobility by using expected shortest path routing. For this, the forwarding metric used is Expected Multi-Destination Delay for Anycast (EMDDA), which is the expected delay in message delivery from a node to the nearest node in the destination node's group. Practical Expected Delay (PED) of a node is a function of the probability of choosing its neighbor node as a relay and the delay between these two nodes. EMDDA of a node is the smallest value of PED from a node to all the members of the destination group. The advantage of such a scheme is that all possible routes to a destination are taken into account instead of a single shortest-path route. The optimization is that if there is a route available immediately (ride on a mobile device which is taking that route at the current instant) when a message arrives to a node, even with the high expected delay, then it is optimal to choose this route instead of waiting for the shortest path route.

Silva, et al. [79] have proposed an anycast routing protocol for DTN using genetic algorithm. Dijkstra's algorithm is used for finding all possible combinations of shortest paths from a source node to a group of destinations. Genetic algorithm is then applied to select an optimal path from this combination of paths. The results show that, the method lowers delivery delay and improves delivery probability.

Receiver Based Forwarding (RBF) scheme is proposed in [80] for anycast routing in DTN. The authors made the assumption that the current network information, such as path length to destination is known to every node in advance. In selecting a relay node, path length from node to receiver together with a number of possible receivers is taken into account. Instead of forwarding through a shortest path, the method selects a path which includes more receivers. Simulation results show that the method achieves better delivery ratio and low end-to end-delay compared to normal forwarding.

\section{Probabilistic}

Xiao, et al. [81] have proposed an anycast routing protocol for probabilistically connected DTN based on a metric called MDRA (Maximum Delivery Rate for Anycast). MDRA is the maximum value of delivery probability for forwarding a message from a node to a group of destinations. Each node computes the MDRA value individually from the contact probability with other nodes. A node with the highest value of MDRA is selected as the relay node to forward the message. Compared to the existing anycast routing protocols, the proposed method increases the delivery ratio and reduces the average delivery delay. The computational overhead is also reduced.

\subsection{Multi- Copy, Unicast, Pure Opportunistic}

\section{Routing Criteria and Metric Based}

The Resource Allocation Protocol for Intentional DTN (RAPID) [82] algorithm assigns different utility values to messages in various situations when different performance metrics are to be optimized. To minimize the average delay, the utility of a message is inversely proportional to its expected delay. To minimize the miss rate, utility of the message is the probability of it reaching in time. To minimize maximum delay, the utility of a message with highest expected delay is the 
negative of its expected delay, and zero for other messages buffered at the same node. In the forwarding algorithm of RAPID, the nodes first share the metadata and then the messages in decreasing order of their utility values. Simulation results show that the average and the maximum data delivery delay in RAPID as compared to MaxProp [30], Spray and Wait [35] and Random forwarding schemes, is significantly lower and the delivery rate is consistently higher.

In the scheme proposed by Shevade, et al. [83], every node in the network calculates three link qualities, namely, the average delivery delay, variance in delivery delay, and average available bandwidth between itself and its neighbors. These are then shared with the neighbors. Having received all the link information, a node has the liberty to choose the routing path for its message to optimize its individual performance. The destination on receiving a packet floods the acknowledgement in the network as a proof of its good behavior. Using simulations, the authors prove that this protocol is able to incorporate cooperation among selfish nodes.

\section{Prediction Based}

Mobility Prediction-based Adaptive Data gathering protocol (MAPD) is proposed in [84] for data gathering in Delay Tolerant Mobile Sensor Networks. On receiving a message, a node forwards copy of the message to nodes, having higher delivery probability, which is the likelihood of meeting the destination. Mobility of the node is predicted using the random waypoint mobility model. An optimization strategy is proposed for queue management in which messages with less survival time are given higher priority for transmission.

\section{Probabilistic Based}

Lindgren, et al. [8] have observed from real mobility traces that nodes in the network, instead of roaming about randomly, exhibit some patterns in their motion. One can predict future contacts with certain probability. Therefore, some prediction value can be assigned to each node for every location or destination. To capture every aspect of delivery probability for some node, a metric named as delivery predictability is introduced in this paper. It records how good a node may act as a forwarder to some other node. The value of delivery predictability changes with every encounter. The metric also considers the degradation in the probability if two nodes have not seen each other in a while. As messages can be delivered transitively, the metric should capture such behavior also, i.e., if A meets B frequently and B meets C frequently, then C may act as a good forwarder for messages with $\mathrm{A}$ as the destination and vice versa. Probabilistic routing considers the value of delivery predictability at some node to make the forwarding decision. If the value of this metric is higher at the encountered node than the node currently carrying the message, only then the message is propagated to it. To evaluate the performance of probabilistic routing, authors have considered two scenarios. The first scenario is based on random motion of the nodes, in which nodes choose both the destination and speed of moving there randomly. This scenario is not exactly in accordance with the practical observation that nodes do not move randomly altogether rather they move to certain places more often. Therefore, a more realistic model, called the "community model", is used. In this model, nodes move to locations with some probability. In both scenarios, PROPHET performs either comparable or better than Epidemic routing [29] depending on the buffer size allocated. For community model, the overhead associated is much less in PROPHET, making it more efficient.

\section{History of Encounters Based}

In [85] a routing protocol for DTN environment, having heterogeneous capabilities and mobility patterns is proposed. The nodes in the network belong to different classes, for instance, vehicles, mobile devices, and sensors, etc. Each node in the network calculates utility for all other nodes using a utility function, which is based on the delivery probability of that node. A node forwards a copy of a message to a relay node which has higher utility value. Similar to the quality metric, the utility function can be destination dependent and destination independent. For destination dependent utility function, node utility values differ for different destinations. For destination independent utility function, node utility value depends on the node's characteristics rather than the destination. Thus, it will be only one for a node. Based on the utility functions used, authors have implemented following three methods. In the Last-Seen-First (LSF) method, a node which most recently encountered the destination is selected as the relay. A node having the highest mobility is selected as the relay in case of the Most-Mobile-First (MMF) method. In Most-Social First (MSF) method, a node having maximum number of social links is selected as the relay. Both MMF and MSF are destination independent methods, whereas LSF is destination dependent. Simulation results show that the above three methods outperform tree-based Spray and Wait method [75] in terms of delivery ratio and end-to-end delivery delay.

Delegation forwarding algorithm proposed by Erramilli, et al. [36] compares the quality of every encountered node with the current level of the message, where level stores the highest value of the quality seen by the message so far. Initially, the level is set to the quality of the source. If a higher quality node is found, the level value is updated to the quality of this node and is forwarded to it. The quality metric can be either destination dependent or independent. E.g., it may depict total number of contacts with other nodes or most recent contact with any node. These are destination 
independent. Total number of contacts with the destination and most recent contact with the destination are examples of destination dependent quality metrics. Based on the quality metric chosen, delegation forwarding is classified into four types - delegation destination frequency, delegation frequency, delegation destination last contact, and delegation last contact. Delegation destination frequency turns out to be the best delegation forwarding scheme as it directly depends on node-destination encounters.).

The paper [86] concerns the data dissemination issue in VANETs (Vehicular Ad-hoc Networks). The problem addressed here is to deliver the message to the nodes in a specific area. Patterns are found in the node's travel paths using historic data, exploiting which nodes are selected as relays. The nodes which have a high probability of travelling to the desired area and in lesser time are selected as the relays. For this purpose, the road map of the region is also used, which is used to find the delivery delay for a message originating at a certain point. The scheme is to choose between carrying in low density regions of the network and relaying in high density regions, where, carrying means that the node having the message keeps the message with itself unless it meets the destination or it goes to a high density region where it relays the message to some other node.

In FRESH (Fresher Encounter Search) [87], all nodes store the time they last encountered each other and a relay node is decided based on most recent interaction with the destination.

Probability Delegation Forwarding (PDF) [88] is an extension of a delegation forwarding scheme discussed in the previous section to further reduce the cost. The authors have proposed to use some probability of forwarding a message together with quality metric to achieve this. When a node A encounters a node B with higher value of the quality metric than level variable in A, the node A will forward the message to node B with a probability p. Here, if a very small value of $p$ is chosen, the delivery latency will increase, because message forwarding will be very much restricted. To combat this, Threshold-based Probability Delegation Forwarding (TPDF) is also proposed in which, over a threshold of difference between qualities of two nodes, the probability would not be taken into consideration. It means if the encountered node is far better a carrier, than the nodes visited so far, the message must be relayed to it. The cost of PDF scheme is less than that of delegation forwarding and for TPDF, with a proper value of threshold; delivery latency similar to delegation forwarding can be achieved.

\section{Using Extra Nodes}

Tariq, et al. [89] have proposed a message ferry route design algorithm for DTN called, Optimized Way Points (OPWP). The network can have a single message ferry, which is a special node that carries the message from source and delivers it to the destination by repeatedly visiting other nodes. On each visit, ferry will meet each node with a certain contact probability. The region where the ferry meets a particular node is called a waypoint, which is calculated based on the contact probability between that node and the ferry. The optimal route to the destination is an ordered set of waypoints and the waiting time at those waypoints of the ferry. The ordering of these waypoints is done in such a way that the route is of minimum length.

Zhao, et al. [90] have proposed a message ferry based approach for routing. A special node, called Message Ferry, carries messages from source to destination by traversing a fixed route. Two schemes have been proposed for message ferrying. The first one is Node Initiated Message Ferrying (NIMF), in which the nodes proactively move towards the ferry. Message ferry traverses in a fixed route and the source and destination of a message are required to meet the ferry whenever they are in need to exchange a message. The second approach is Ferry Initiated Message Ferrying (FIMF), in which, the message ferry proactively moves to the source and destination to exchange a message. As compared to Epidemic routing, message ferrying performs better in terms of both cost and data delivery rate. This is due to the fact that the data delivery rate in Epidemic routing depends very much on node mobility unlike the ferry based schemes as the later is proactive in nature.

\section{Flooding Based}

This is one of the first schemes for routing in DTN environment, proposed by Vahdat, et al. [29]. It is a simple flooding based forwarding approach where the source node copies its message to every node it meets and then these nodes do the same. This scheme is based on the idea that the message will eventually find its destination through transitive exchanges between nodes, if they are spread across the connected portion of the network. Epidemic routing protocol can achieve a delivery rate of $100 \%$ in partitioned networks where ad hoc routing protocols fail entirely. But flooding results in high resource consumption in terms of both power and storage. For reducing the resources consumed while routing the message from source to destination, a limit on either the message hop count or the buffer space allocated at each node can be put. Therefore, the message will be dropped after its TTL (Time-To-Live) expires or if the buffer at the peer is full. By setting a low value of hop count, the message delivery ratio will decrease and average delivery time will increase. On the other hand, if hop count is high, messages will be dispersed in the network more quickly, resulting in a higher delivery ratio and lower average delivery time. Thus a trade-off has to be made between these performance metrics. 
In another flooding based scheme, proposed by Spyropoulos, et al.[35], the source node replicates the message into the first ' $L$ ' nodes it encounters. This phase is called the spray phase, where limited flooding takes place. If the destination is not one of these ' $L$ ' nodes, then it will directly receive a copy of the message from one or more of these ' $L$ ' nodes. This is the wait phase of the algorithm. Thus, spray and wait protocol is a combination of controlled epidemic routing (spray phase) and direct transmissions (wait phase). Another variation of the algorithm works following a binary distribution pattern. E.g., if a node A has $n$ logical copies of a message and it encounters a node B, then A sprays $n / 2$ copies to B and keeps the remaining copies with itself. The process is repeated until A is left with just a single copy when it switches to direct transmission. This variation is called Binary Spray and Wait. Spray and wait is a ticket based forwarding scheme, in which, every message is assigned logical tickets. Tickets depict the number of relays the message is to be forwarded to. The authors have compared their scheme with five other schemes, namely - Epidemic routing [29], Randomized flooding, PROPHET [8], Seek and Focus, and Oracle-based [28] optimal routing schemes. The delivery delay is almost equal to epidemic routing, but much less than other multi-copy algorithms. The total number of transmissions is also lesser as compared to existing protocols.

Spyropoulos, et al. [91] has proposed multi-copy routing protocol for DTN using the concept of spray and wait. Stochastic mobility model is used to model the mobility of nodes in the network. The main idea of the spray and wait protocol [35] is that a pre-defined number of copies of messages are carried by different relay nodes in a way that, at least one of them reaches the destination. In the proposed method, authors follow the same strategy for spraying the message and in the forwarding phase, each relay node is selected based on the utility value of the node which is defined in [75].

LocalCom [92] is an epidemic forwarding scheme developed for DTN based on community structure. Similarity metric is used for forwarding decision, which is calculated from the encounter history of nodes. A node calculates its relationship with each of its neighbors based on similarity. Based on the similarity metric, a contact graph is created. The node with largest degree is selected as an initiator to detect a community, where degree of a node is the sum of weights of all incoming links to that node. Based on the detected community structure, a message is directly forwarded to destination in case of intra community routing. Controlled flooding is used for inter-community routing, where bridge nodes will perform the forwarding between communities. Simulation results show that LocalCom achieves a higher delivery ratio, but overhead, in terms of number of packets forwarded is also increased as compared to PROPHET [8] and Bubble Rap [32].

Adaptive forwarding scheme is proposed in [93] for routing in DTN, with an idea of controlling the replication of message copies. The probability of replicating a message is based on the information about existing copies of the same message in the network. The replication probability decreases with an increase in the number of existing message copies. When two nodes meet each other, they exchange the information about their message copies, which can be used to infer the replication probability. By adaptively adjusting the replication probability, network resource overhead is reduced. Simulation results show that the proposed method produces less number of message copies in the network as compared to the epidemic routing [29] scheme, even though delivery delay is slightly higher.

Delay-differentiated gossiping [94] provides an upper bound on the delivery delay in DTN. Packets are grouped into different classes based on their expected delivery delays. Every message is associated with a probability (which is inversely proportional to expected delivery delay) and time-to-live (TTL) value. On receiving a packet, a node forwards it to all the nodes it encounters based on the probability associated with the packet and its TTL value. Simulation results show that the proposed method achieves deadline assurance close to the desired probability of assurance.

\subsection{Multi- Copy, Multicast, Pure Opportunistic}

\section{Coding Based}

In [95], the performance of a multicast protocol with Random Linear Coding (RLC) is analyzed and compared with simple custodial multicast protocol. The authors have observed that the proposed method yields better performance when the destination group is large and the in-node storage size is small.

\section{Probabilistic Based}

Burgess, et al. [30] have proposed a protocol for vehicular DTNs called MaxProp. For this, their idea is to prioritize the schedule of message delivery to other nodes as well as the schedule of message drop. The buffer is logically divided into two parts with a threshold $t$ on the hop count. The messages having remaining hop count less than $t$ are sorted according to the hop count and packets having remaining hop count greater than $\mathrm{t}$ are sorted according to their delivery probabilities. Delivery probabilities, initialized to the value $1 /(\mathrm{N}-1)$ (where, $\mathrm{N}$ is network size), are calculated through incremental averaging methods (incremented by 1 ) on every encounter and renormalizing it. The messages already stored in a node's buffer are not sent to it again, which is checked with the help of message's hop list. Whenever there is a contact opportunity between two nodes, the messages with destination address as the encountered node are delivered. 
Acknowledgements are sent about the delivered data throughout the network so as to clear the buffers storing those messages. The delivery probability vectors are exchanged. Messages with high hop count are given priority and copied to another node's buffer. The remaining messages, which have not yet been copied to another node's buffer, are sent in the order of their delivery probabilities, with highest probability being first. Authors have compared MaxProp with Oraclebased Dijkstra [28] and ME/DLE (Most Encountered/ Drop Least Encountered) [73] protocols Simulation shows that MaxProp outperforms these schemes in terms of both delivery ratio and delivery latency.

\section{History of Encounters Based}

RelayCast [96] is a mobility assisted multicast routing protocol for DTN. The method is an extension of 2-hop relay routing [66] for multicast. Routing is performed in two phases. In the relay phase, a source node forwards the message to a relay node (or destination) in contact. In the delivery phase, the relay node keeps the message in a separate queue for each destination and forwards the message to a destination, if the destination has not received the message earlier. Simulation results show that RelayCast achieves better throughput and scalability with acceptable delivery delay.

\section{Hierarchical Routing}

A DTN may be densely connected at some points and may be sparsely connected at the others. So, a multicast scheme is proposed in [97], which focuses on varying node densities in DTN and leverages information about the current location and velocity of nodes. In this proposed CAMR scheme, nodes decide whether they belong to the sparse part of the network or the dense part, by finding the mean of the total neighbors they have. If they have, on an average, more than a threshold number of neighbors, then they belong to the denser region of the network and vice-versa. Thus, for unreachable nodes (nodes at greater than 2-hop distance), multicast tree is formed rooted at the source, to all the destinations and messages are sent along the downstream path.

\section{Pure Opportunistic Data Dissemination in DTN}

Pure opportunistic data dissemination schemes can be of two types - reactive and proactive.

\subsection{Reactive Methods}

\section{(Using) Common Nodes}

Zyba, et al. [98] classified mobile users based on their social behavior, for data dissemination in opportunistic mobile networks. The mobile traces are divided into multiple areas and the users are classified into socials and vagabonds. Socials are devices, which visited the geographical area more periodically or frequently, whereas vagabonds are not as frequent visitors as socials. Authors used socials and vagabonds for data dissemination, while the existing techniques consider only socials. Least-total appearance, Fourier, and Binning are the three methods that are used to classify users into socials and vagabonds. Experiments on real datasets, such as San Francisco [99], Dartmouth [100], and Second Life [101][102], show that vagabonds are more effective in data dissemination compared to socials, in the scenario of large populations.

\section{Popularity of Data Items}

CEDO [103] is a content-centric data dissemination system in which every node caches data items ranked by their popularity. Requesters move through the DTN in search of a content which might be present in the cache of one or more peer nodes they will encounter. Successful data dissemination is achieved, if the content is obtained from a requesting node before the expiry of the request timeout value. The Success rate of data dissemination depends on the number of available replicas of a data item, which is constrained by the cache size of nodes and popularity of individual items. CEDO defines delivery rate metric for each data item, as the number of replicas of the item successfully delivered to requesters in unit time. Their objective is to maximize the aggregate delivery rate of all data items in all requests and they propose some optimal policies to be followed, by each node while caching or dropping data items.

\section{Prediction Based}

The authors in [104] have developed a content sharing scheme for delay tolerant smart phone network based on the concept of discover-predict-deliver (DPD) strategy. The DPD scheme focuses on data dissemination in indoor environments. Content discovery and delivery leverages the human mobility patterns, which are known to be regular and predictable. Content requesters forward their interests and mobility patterns to one-hop nodes, which act as request carriers. Spreading of content requests in space and time is controlled by the requestor, which specifies the intended number of replicas and its lifetime. Content query forwarding decision depends on the ratio of content discovery versus delivery times and the physical distance between the current request-carrier and the requestor. Current location of the requestor is obtained by prediction, based on its pre-announced mobility pattern. Upon discovery of a requested content, 
the number of matching contents actually forwarded to the requestor, are limited using some pre-specified threshold. Content forwarding relays are selected based on a utility function, which considers mobility information of the relay nodes as well as the requestor. Nodes which contact the requesting node earlier and stay closer for longer duration are chosen as relays. Experiments are conducted on real DTN test bed considering humans carrying smart-phones and the results show that DPD can successfully discover and deliver $87 \%$ of the contents within 2 hours as long as the contents are available in $30 \%$ of the nodes.

\section{Coding Based}

A data dissemination scheme based on the content characteristics is proposed in [105]. Content can be a file, video or Web-content. Three message scheduling algorithms were proposed as an extension to Hybrid Erasure Coding (HEC) scheme [106] for content delivery. The algorithms are Sequential Forwarding (SF), Full Interleaving (FI), and Block-based Interleaving (BI). Each message is split into erasure code blocks. The first block is directly transmitted to the relay node and the second block is transmitted using one of the aforementioned message scheduling algorithms. SF algorithm sends code blocks according to the order of the messages. Using FI algorithm, interleaving of code blocks from different messages is done. In case of the BI algorithm, sending processes are interleaved. Layered Multiple Description Coding (LMDC) is used for data dissemination, in which the data are layered across multiple messages with multiple descriptions. Simulation results show that SF and BI algorithms are more effective when there is better network connectivity, while the FI algorithm performs well in case of poor connectivity.

\subsection{Proactive Methods}

PodNet [107] is Pub/Sub based middleware architecture for data dissemination system in opportunistic network of mobile phones. There is no network layer in PodNet; instead contents are disseminated opportunistically by the mobile nodes. Receiver-driven solicitation protocol and node mobility determines the content distribution. Contents are organized in a hierarchical structure and grouped into feeds. Each feed, consists of a number of entries from a peer for an interested data object. The contents are shared using a solicitation protocol, which uses a pull based approach. Pull based approach is a receiver driven method, in which sender only delivers the content based on the request and the receiver have control over the content.

\section{Open Problems}

In previous sections, we have discussed about the state-of-the art of DTN routing and data dissemination protocols comprising of both social and pure opportunistic schemes. As part of it, we have observed some of the challenges and open problems, which are yet to be addressed. We summarize them here for the benefit of future researchers.

- Lack of general framework and mathematical modeling: There is no generalized framework for defining network models, analyzing mobility and network performance for DTN routing protocols. Instead, each routing protocol chooses own network and mobility models according to the applications they aim to support. In the absence of any standard model, authors just compare their proposed routing protocols with the naive flooding based schemes. Since resource constraints and delay requirements vary across different applications of DTN, mathematically modeling DTN is a challenging problem.

- Incorporating new technologies for optimizing routing decision: Since the selection of forwarding metric is based on heuristics, concepts like artificial intelligence, fuzzy logic, and genetic algorithm [79], etc., can be incorporated in taking routing decisions.

- Multiple forwarding metrics: Instead of using single metric, combining metrics for social-based and pure opportunistic routing will be exciting to investigate.

- Loop-free algorithms: Many of the algorithms perform routing by computing the maximum value of the forwarding metric locally. The drawback associated with this approach is that, the message may get stuck on the node having the maximum value and will not be forwarded to the destination. Currently, there are no mechanisms to prevent routing loops in DTN.

- Efficient buffer management: Most of the algorithms make an idealistic assumption that nodes have infinite buffer and hence, no replacement policy is required. Also, they do not consider fixing schedules for packet transmission and dropping. Cooperative caching exists for wireless networks, but it is difficult in DTN, because of the lack of connectivity and difficulty in consistency maintenance between multiple message copies. Although some efforts for cooperative caching [108] exists in DTN, investigating the effects of social relations between nodes on caching can be interesting. Also prediction of buffer space is also an important aspect to look for, since buffer space is limited in relay node, especially when the relay node is a smart phone.

- Scheduling of message transmission: Researchers have to think about how to perform optimal transmission scheduling in DTN under the constraints of storage and energy. Only a few works, e.g. [109] exist to handle such 
issues.

- Quality of Service (QoS): Not many QoS metrics have been incorporated into DTN routing protocols. Some authors [94] have considered providing deadline-based assurance for message delivery in DTN. However, compared to wireless networks, providing QoS guarantees in DTN is still an open area.

- Finding new applications of DTN in NDN and Internet of Things (IoT): Content Centric Network (CCN) and Named Data Network (NDN) are the proposed future Internet architectures. NDN provides inherent support for DTN applications. Researchers should look into the possibilities of developing DTN applications over NDN architecture. Similarly, a new networking paradigm to interconnect physical world smart entities is Internet of Things (IoT). Building IoT over DTN is possible in case of limited connectivity.

- Security aspects of routing: Most of the routing protocols do not consider the security aspects. Since the nodes in DTN are routers (act as a relay), possibility of attacks, such as Denial of Services (DoS), spoofing, and presence of a malicious node is to be considered. A detailed description of DTN security issues and challenges has been given in [110].

- Developing intelligent routing protocols: Most of the DTN routing protocols are application specific. Intelligent protocols have to be designed, such that nodes can adaptively adjust the traffic rates based on the type of communication. The Self-learning ability of routing protocols can be a future area of research.

- Evolutionary community detection algorithms: There exist plenty of community detection algorithms in literature; most of them are static, which cannot be directly applied in DTN. But only few dynamic community detection algorithms are currently applied to routing in DTN. Development of new dynamic community detection algorithm, to identify the evolution of communities, will help to improve DTN routing protocols.

- Naming and addressing problem: The addressing part of DTN can adopt some features from new information centric network architectures like NDN. Instead of late binding of data packets to IP address as in DTN, named data objects can be directly used for delivery. Further research can be focused on how this can be done. Incorporating information centric network architecture in DTN is proposed in [111].

- Selfish behavior of nodes: Most of the routing protocols consider that, all the nodes in DTN participate equally and honestly in the process of forwarding a message to the destination. But there are some protocols [46][52][83] which consider that nodes are selfish, because they do not want to spend energy for message forwarding for others. Therefore, some incentive based methods are developed, but the area is still open for further research.

- Few social-based multicast protocols: While many social-based unicast protocols exist in literature, only few social-based multicast protocols are proposed. Gao, et al. [53] proposed first social-based multicast routing protocol for DTN. Recently, Deng, et al. [112] have proposed a social similarity based multicast routing protocol for DTN. But to the best of our knowledge, there is no social-based single copy multicast protocol developed for DTN.

\section{Conclusion}

DTNs allow a node to store and carry a message until a suitable relay node is found to hand it over. Many existing surveys have covered several aspects of DTN routing protocols in depth. However, the relation between message replication (to approach the destination node(s) through different available forwarding ways) and single or group communication primitives (unicast, multicast, and anycast based on the number of recipients) adopted have not been studied so far. Moreover, the recent growth in using DTN for content sharing between peers has also not been studied by any existing survey. In this paper, we have discussed the adaptability of DTN features in emerging areas like ICN, NDN, IoT, etc. We have introduced a novel taxonomy considering message replication and network primitives used in social-based and pure opportunistic DTN routing protocols. We have also studied social-based and pure opportunistic data dissemination protocols in DTN. Finally, a set of open challenges has been listed to provide directions to the prospective researchers.

\section{Acknowledgment}

This work is partially supported by the following grants: MHRD (GoI) FIG (A) 100579-ECD and DST (GoI) SB/FTP/ ETA- 23/2013.

\section{References}

[1] C. Boldrini, M. Conti, and A. Passarella, "Context and resource awareness in opportunistic network data dissemination," In Proceedings of IEEE International Symposium on World of Wireless Mobile and Multimedia Networks (WoWMoM), 2008, pp. 1-6.

[2] P. Juang, H. Oki, Y. Wang, M. Martonosi, S.L Peh, and D. Rubenstein, "Energy-efficient computing for wildlife tracking: Design tradeoffs and early experiences with ZebraNet," In ACM Sigplan Notices, 37 (10) (2002), pp. 96-107.

[3] UMassDieselNet: A Bus-based Disruption Tolerant Network, <http://prisms.cs.umass.edu/diesel/> [accessed on 19 March, 2015].

[4] P. Hui, A. Chaintreau, J. Scott, R. Gass, J. Crowcroft, and C. Diot, "Pocket switched networks and human mobility in conference environments," In Proceedings of ACM Special Interest Group on Data Communication (SIGCOMM) workshop on Delay-tolerant networking, 2005, pp. 244-251.

[5] L. Franck, "Performance comparison of mobile ad-hoc and delay tolerant networks," <www-public.int-evry.fr > [accessed on 23 March, 
$2015]$.

[6] C. Perkins, E. Belding-Royer, and S. Das, “Ad hoc On-Demand Distance Vector (AODV) Routing,” RFC 3561, IETF Network Working Group, July, 2003.

[7] Johnson, B. David and D.A. Maltz, "Dynamic Source Routing in Ad Hoc Wireless Networks," In Mobile Computing, 1996 , pp. 153-181.

[8] A. Lindgren, A. Doria, and O. Schelen, "Probabilistic routing in intermittently connected networks," In ACM SIGMOBILE mobile computing and communications review, 7 (3) (2003), pp. 19-20.

[9] Z. Zhang, "Routing in intermittently connected mobile ad hoc networks and delay tolerant networks: Overview and challenges," In IEEE Communication Surveys Tutorials, 8 (1) (2006), pp. 24-37.

[10] Y. Zhu, B. Xu, X. Shi, andY. Wang, “A survey of social-based routing in delay tolerant networks: Positive and negative social effects," In IEEE Communication Surveys Tutorials, 14 (3) (2012), pp. 1-15.

[11] K. Wei, X. Liang and K. Xu. "A survey of social-aware routing protocols in delay tolerant networks: applications, taxonomy and designrelated issues," In IEEE Communications Surveys and Tutorials, 16 (1) (2014), pp. 556-578.

[12] Y. Cao and Z. Sun, "Routing in delay/disruption tolerant networks: A taxonomy, survey and challenges," In IEEE Communications Surveys and Tutorials, 15 (2) (2013), pp. 654-677.

[13] M. J. Khabbaz, C. M. Assi and W. F. Fawaz, "Disruption-Tolerant Networking: A Comprehensive Survey on Recent Developments and Persisting Challenges," In IEEE Communications Surveys and Tutorials, 14 (2) (2012), pp. 607-640.

[14] I. Psaras, L. Wood, and R. Tafazolli, "Delay-/disruption-tolerant networking: State of the art and future challenges," $<$ http:// ee. ucl. ac. uk/ uceeips/dtn-srv-ipsaras. pdf $>$ [accessed on 17 March, 2015].

[15] G. Tyson, J. Bigham, and E. Bodanese, "Towards an information-centric delay-tolerant network," In Proceedings of IEEE Conference on Computer Communications Workshops (INFOCOM WKSHPS), 2013, pp. 387-392.

[16] E. Nordstrom, P. Gunningberg, and C. Rohner, "Haggle: Relevance-Aware Content Sharing for Mobile Devices Using Search, 2012," $<$ http:// haggle. googlecode. com $>$ [accessed on 20 March, 2015].

[17] E. Monticelli, M. Arumaithurai, I. Psaras, X. Fu, and K. K. Ramakrishnan, "Combining Opportunistic and Information Centric Networks in Real World Applications," <https://www.netsys2015.com/wp-content/uploads/NetSys2015_PhD-Forum_Monticelli.pdf > [accessed on 21 March, 2015].

[18] C. Anastasiades, A. Uruqi, and T. Braun, "Content discovery in opportunistic content-centric networks," In Proceedings of IEEE 37th Conference on Local Computer Networks Workshops (LCN Workshops), 2012, pp. 1044-1052.

[19] C. Anastasiades, T. Schmid, J. Weber, and T. Braun, "Opportunistic content-centric data transmission during short network contacts," In Proceedings of IEEE Wireless Communications and Networking Conference (WCNC), 2014, pp. 2516-2521.

[20] Y. T. Yu, C. Tandiono, X. Li, Y. Lu, M. Y. Sanadidi, and M. Gerla, "ICAN: Information-centric context-aware ad-hoc network," In Proceedings of International Conference on Computing, Networking and Communications (ICNC), 2014, pp. 578-582.

[21] B. Batista, and P. Mendes, "ICON-An Information Centric Architecture for Opportunistic Networks," In Proceedings of IEEE INFOCOM Workshop on Emerging Design Choices in Name-Oriented Networking, 2013.

[22] J. Wang, R. Wakikawa, and L. Zhang, "DMND: Collecting data from mobiles using named data," In Proceedings of IEEE Vehicular Networking Conference (VNC), 2010, pp. 49-56.

[23] G. Grassi, D. Pesavento, G. Pau, R. Vuyyuru, R. Wakikawa, and L. Zhang, "VANET via Named Data Networking," In Proceedings of IEEE Conference on Computer Communication Workshops (INFOCOM WKSHPS), 2014, pp. 410-415.

[24] T. Ogawara, Y. Kawahara, and T. Asami, "Information dissemination performance of a disaster-tolerant ndn-based distributed application in disrupted cellular networks," In Proceedings of 13th IEEE International Conference on Peer-to-Peer Computing (P2P), 2013, pp. 1-5.

[25] M. C. Domingo, "An overview of the internet of underwater things," Network and Computer Applications, 35(6) (2012), pp.1879-1890.

[26] B. Guo, D. Zhang, Z. Wang, Z. Yu, and X. Zhou, "Opportunistic IoT: exploring the harmonious interaction between human and the internet of things," In Journal of Network and Computer Applications, 36 (6) (2013), pp.1531-1539.

[27] F. M. Al-Turjman, A. E. Al-Fagih, W. M. Alsalih, and H. S. Hassanein, "A delay-tolerant framework for integrated RSNs in IoT," In Computer Communications, 36 (9) (2013), pp. 998-1010.

[28] S. Jain, K. Fall, and R. Patra, "Routing in a delay tolerant network," In Proceedings of ACM Special Interest Group on Data Communication (SIGCOMM), Vol. 34, No. 4, 2004, pp. 145-158.

[29] A. Vahdat and D. Becker, "Epidemic routing for partially connected ad hoc networks," Technical Report CS-200006, Duke University, 2000.

[30] J. Burgess, B. Gallagher, D. Jensen, and B.N. Levine, "MaxProp: Routing for Vehicle-Based Disruption-Tolerant Networks," In Proceedings of IEEE International Conference on Computer Communications (INFOCOM), Vol. 6, 2006, pp. 1-11.

[31] E.M. Daly and M. Haahr, "Social network analysis for routing in disconnected delay-tolerant MANET's," In Proceedings of the 8th ACM International Symposium on Mobile Ad hoc Networking and Computing (MobiHoc), 2007, pp. 32-40.

[32] P. Hui, J. Crowcroft, and E. Yoneki, "Bubble Rap: Social-based forwarding in delay-tolerant networks," In IEEE Transactions on Mobile Computing, 10 (11) (2011), pp. 1576-1589.

[33] W. Chen, R. K. Guha, T. J. Kwon, J. Lee, and Y. Y Hsu, "A survey and challenges in routing and data dissemination in vehicular ad hoc networks," In Wireless Communications and Mobile Computing, 11 (7) (2011), pp. 787-795.

[34] N. Benamar, K. D. Singh, M. Benamar, D.EI Ouadghiri, and J.M. Bonnin, "Routing protocols in Vehicular Delay Tolerant Networks: A comprehensive survey," In Computer Communications, 48 (2014), pp. 141-158.

[35] T. Spyropoulos, K. Psounis, and C. S. Raghavendra, "Spray and Wait: An Efficient Routing scheme for Intermittently Connected Mobile Networks," In Proceedings of ACM Special Interest Group on Data Communication (SIGCOMM) workshop on Delay-tolerant networking, 2005, pp. 252-259.

[36] V. Erramilli, M. Crovella, A. Chaintreau, and C. Diot. "Delegation Forwarding", In Proceedings of Ninth ACM International Symposium on Mobile ad hoc networking and computing (MobiHoc), 2008, pp. 251-260.

[37] A. Mtibaa, M. May, C. Diot, and M. Ammar, "Peoplerank: Social opportunistic forwarding," In Proceedings of IEEE International Conference on Computer Communications (INFOCOM), 2010, pp. 1-5.

[38] S. Brin and L. Page, "The anatomy of a large-scale hypertextual Web search engine," In Computer networks and ISDN systems, 30(1) (1998), pp. 107-117.

[39] K. Jahanbakhsh, G. C. Shoja, and V. King, "Social-greedy: a socially-based greedy routing algorithm for delay tolerant networks," In Proceedings of Second International Workshop on Mobile Opportunistic Networking, 2010, pp. 159-162.

[40] E. Bulut and B. K. Szymansk, "Exploiting friendship relations for efficient routing in mobile social networks," In IEEE Transactions on Parallel and Distributed Systems, 23 (2012), pp. 2254-2265. 
[41] D. Zhang, Z. Wang, B. Guo, X. Zhou, and V. Raychoudhury, "A dynamic community creation mechanism in opportunistic mobile social networks," In Proceedings of Third IEEE International conference on Privacy, Security, Risk and Trust (PASSAT), Third IEEE international conference on Social Computing (SOCIALCOM), 2011, pp. 509-514.

[42] P. Hui and J. Crowcroft, "How small labels create big improvements," In Proceedings of Fifth Annual IEEE International Conference on Pervasive Computing and Communications Workshops (PerCom Workshops' 07), 2007, pp. 65-70.

[43] A. Mei, G. Morabito, P. Santi, and J. Stefa, "Social-aware stateless forwarding in pocket switched networks," In Proceedings of IEEE International Conference on Computer Communications (INFOCOM), 2011, pp. 251-255.

[44] F. Fabbri and Verdone, “A sociability-based routing scheme for delay-tolerant networks,” In EURASIP Journal on Wireless Communications and Networking, 1 (2011).

[45] T. Abdelkader, K. Naik, A. N. Nayak, and V. Srivastava, "SGBR: A routing protocol for delay tolerant networks using social grouping," In IEEE Transactions on Parallel and Distributed Systems, 24(12) (2013), pp. 2472-2481.

[46] Q. Li, W. Gao, S. Zhu, and G. Cao, "A routing protocol for socially selfish delay tolerant networks," InAd Hoc Networks, 10(8) (2012), pp. 1619-1632.

[47] S. Chen, J. Zhang, and Q. Gao, "Social-based Hierarchical Routing in Delay-tolerant Networks,". In Journal of Computation System, 9(16) (2012), pp.4881-4888.

[48] K. Chen and H. Shen, "Smart: Lightweight distributed social map based routing in delay tolerant networks," In 20th IEEE International Conference on Network Protocols (ICNP), 2012, pp. 1-10.

[49] T. Zhou, R. R. Choudhury, and K. Chakrabarty, "Diverse routing: Exploiting social behavior for routing in delay-tolerant networks," In Proceedings of International Conference on Computational Science and Engineering (CSE), Vol. 4, 2009, pp. 1115-1122.

[50] L. Zhao, F. Li, C. Zhang, and Y. Wang, "Routing with multi-level social groups in mobile opportunistic networks," In Proceedings of IEEE Global Communications Conference (GLOBECOM), 2012, pp. 5290-5295.

[51] J. Scott, R. Gass, J. Crowcroft, P. Hui, C. Diot and A. Chaintreau, "CRAWDAD trace Cambridge/haggle/imote/Cambridge (v. 2006-01-31)," $<$ http://crawdad. cs. dartmouth. edu/cambridge/haggle/imote/cambridge> [accessed on 25 March, 2015].

[52] A. Mei and J. Stefa, "Give2get: Forwarding in social mobile wireless networks of selfish individuals," In IEEE Transactions on Dependable and Secure Computing, 9(4) (2012), pp. 569-582.

[53] W. Gao, Q. Li, B. Zhao, and G. Cao, "Multicasting in delay tolerant networks: a social network perspective," In Proceedings of 10th ACM International Symposium on Mobile Ad hoc Networking and Computing (MobiHoc), 2009, pp. 299-308.

[54] F. Li, C. Zhang, Z. Gao, L. Zhao, and Y. Wang, "Social feature enhanced group-based routing for wireless delay tolerant networks," In proceedings of 8th IEEE International Conference on Mobile Ad-hoc and Sensor Networks (MSN), 2012, pp. 68-74.

[55] E. Yoneki, P. Hui, S. Y. Chan and J. Crowcroft, "A socio-aware overlay for publish/subscribe communication in delay tolerant networks," In Proceedings of 10th ACM Symposium on Modeling, Analysis, and Simulation of Wireless and Mobile Systems (MSWiM), 2007, pp. 225234.

[56] F. Li and J. Wu, "Mops: Providing content-based service in disruption-tolerant networks," In 29th IEEE International Conference on Distributed Computing Systems, 2009, pp. 526-533.

[57] P. Costa, C. Mascolo and M. Musolesi. "Socially-aware routing for publish-subscribe in delay-tolerant mobile ad hoc networks," In IEEE Journal on Selected Areas in Communications, 26(5) (2008), pp. 748-760.

[58] W. Gao and G. Cao, "User-centric data dissemination in disruption tolerant networks," In Proceedings of IEEE International Conference on Computer Communications (INFOCOM), 2011, pp. 3119-3127.

[59] C. Boldrini, M. Conti, and A. Passarella, "Contentplace: social-aware data dissemination in opportunistic networks," In Proceedings of ACM 11th International Symposium on Modelling, Analysis and Simulation of Wireless and Mobile Systems (MSWiM), 2008, pp. 203-210.

[60] B. Guo, Z. Yu, X. Zhou, and D. Zhang, "Hybrid SN: interlinking opportunistic and online communities to augment information dissemination," In Proceedings of 9th IEEE International Conference on Ubiquitous Intelligence \& Computing and 9th International Conference on Autonomic \& Trusted Computing (UIC/ATC), 2012, pp. 188-195.

[61] M. Conti, F. Delmastro, and A. Passarella, "Social-aware Content Sharing in Opportunistic Networks," In Proceedings of 6th Annual IEEE Communications Society Conference on Sensor, Mesh and Ad Hoc Communications and Networks Workshops (SECON Workshops '09), 2009, pp.1-3.

[62] C. Boldrini, M. Conti, and A. Passarella, "Modelling data dissemination in opportunistic networks," In Proceedings of 3rd ACM workshop on Challenged networks, 2008, pp. 89-96.

[63] K. Tan, Q. Zhang, and W. Zhu, "Shortest path routing in partially connected ad hoc networks," In Proceedings of IEEE Global Telecommunications Conference (GLOBECOM), Vol. 2, 2003, pp. 1038-1042.

[64] J. LeBrun, C. C. Chuah, D. Ghosal and M. Zhang, "Knowledge-Based Opportunistic Forwarding in Vehicular Wireless Ad Hoc Networks," In Proceedings of IEEE Vehicular Technology Conference (VTC), Vol. 4, 2005, pp. 2289-2293.

[65] V. Conan, J. Leguay, and T. Friedman, "Fixed point opportunistic routing in delay tolerant networks," In IEEE Journal on Selected Areas in Communications, 26(5) (2008), pp. 773-782.

[66] M. Grossglauser and D. Tse, "Mobility increases the capacity of ad-hoc wireless networks," In Proceedings of 12th Annual Joint Conference of the IEEE Computer and Communications Societies (INFOCOM), Vol. 3, 2001, pp. 1360-1369.

[67] J. Leguay, T. Friedman, V. Conan, "Evaluating Mobility Pattern Space Routing for DTNs," In Proceedings of 25th IEEE International Conference on Computer Communications (INFOCOM), 2006, pp.1-10.

[68] M. Musolesi and C. Mascolo, "CAR: Context-aware adaptive routing for delay-tolerant mobile networks," In IEEE Transactions on Mobile Computing, 8(2) 2009, pp. 246-260.

[69] Q. Yuan, I. Cardei, and J. Wu, "Predict and relay: an efficient routing in disruption-tolerant networks," In Proceedings of 10th ACM international symposium on Mobile ad hoc networking and computing, 2009, pp. 95-104.

[70] Z. Guo, B. Wang, and J.H. Cui, "Prediction assisted single-copy routing in underwater delay tolerant networks," In Proceedings of IEEE Global Telecommunications Conference (GLOBECOM), 2010, pp. 1-6.

[71] N. Sarafijanovic-Djukic, M. Piorkowski, and M. Grossglauser, "Island hopping: Efficient mobility-assisted forwarding in partitioned networks," In Proceedings of 3rd Annual IEEE Communications Society on Sensor and Ad Hoc Communications and Networks (SECON), Vol. 1, 2006, pp. 226-235.

[72] C. Liu and J. Wu, "An Optimal Probabilistic Forwarding Protocol in Delay Tolerant Networks," In Proceedings of 10th ACM Internationa Symposium on Mobile ad hoc networking and Computing (MobiHoc), 2009, pp. 105-114.

[73] B. Burns, O. Brock, and B. N. Levine, "MV routing and capacity building in disruption tolerant networks," In Proceedings of 24th Annual 
Joint Conference of the IEEE Computer and Communications Societies (INFOCOM), 2005, pp.398-408.

[74] Y. Dai, P. Yang, G. Chen, and J. Wu, "Cfp: Integration of fountain codes and optimal probabilistic forwarding in dtns" In Proceedings of IEEE Global Telecommunications Conference (GLOBECOM), 2010, pp. 1-5.

[75] T. Spyropoulos, K. Psounis, and C. S. Raghavendra, "Efficient routing in intermittently connected mobile networks: The single-copy case," In IEEE/ACM Transactions on Networking (TON), 16 (1) (2008), pp. 63-76.

[76] J. Wu and Y. Wang, "A non-replication multicasting scheme in delay tolerant networks," In Proceedings of 7th IEEE International Conference Mobile Adhoc and Sensor Systems (MASS), 2010, pp. 89-98.

[77] Y. Wang, L. Xiaoguang, and J. Wu. "Multicasting in Delay Tolerant Networks: Delegation Forwarding," In Proceedings of IEEE Global Telecommunications Conference (GLOBECOM), 2010, pp. 1-5.

[78] Y. Gong, Y. Xiong, Q. Zhang, Z. Zhang, W. Wang, and Z. Xu, “Anycast routing in delay tolerant networks," In Proceedings of IEEE Global Telecommunications Conference (GLOBECOM), 2006, pp. 1-5.

[79] R. Silva and P. R Guardieiro, "An efficient genetic algorithm for anycast routing in delay/disruption tolerant networks," In Communications Letters, IEEE, 14 (4) (2010), pp. 315-317.

[80] F. Fazl-e-Hadi, N. Shah, A. H. Syed, and M. Yasin, "Adaptive anycast: A new anycast protocol for performance improvement in delay tolerant networks," In Proceedings of IEEE International Conference on Integration Technology (ICIT), 2007, pp. 185-189.

[81] M. Xiao, L. Huang, A. Liu, and W. Chen, "Anycast routing in probabilistically contacted delay tolerant networks," In Proceedings of International Conference on Communications and Mobile Computing (CMC), Vol. 3, 2010, pp. 442-446.

[82] A. Balasubramanian, B. Levine, and A. Venkataramani, "DTN routing as a resource allocation problem," In ACM SIGCOMM Computer Communication Review, 37 (4) (2007), pp. 373-384.

[83] U. Shevade, H. H. Song L. Qiu and Y. Zhang, "Incentive-Aware Routing in DTNs," In Proceedings of IEEE International Conference on Network Protocols (ICNP), 2008, pp. 238-247.

[84] J. Zhu, J. Cao, M. Liu, Y. Zheng, H. Gong, and G. Chen, "A mobility prediction-based adaptive data gathering protocol for delay tolerant mobile sensor network,” In Proceedings of IEEE Global Telecommunications Conference (GLOBECOM), 2008, pp. 1-5.

[85] T. Spyropoulos, T. Turletti, and K. Obraczka, "Routing in delay-tolerant networks comprising heterogeneous node populations," In IEEE Transactions on Mobile Computing, 8 (8) (2009), pp. 1132-1147.

[86] S. Hosseininezhad and V. C. M. Leung, "Data Dissemination for Delay Tolerant Vehicular Networks: Using Historical Mobility Patterns," In Proceedings of 3rd ACM international symposium on Design and Analysis of Intelligent Vehicular Networks and Applications (DIVANET), 2013, pp. 115-122.

[87] H. Dubois-Ferriere, M. Grossglauser, and M. Vetterli, "Age matters: efficient route discovery in mobile ad hoc networks using encounter ages," In Proceedings of 4th ACM International Symposium on Mobile Ad hoc Networking \& Computing (MobiHoc), 2003, pp. $257-266$.

[88] X. Chen, J. Shen, T. Groves, and J. Wu. "Probability Delegation Forwarding in Delay Tolerant Networks," In Proceedings of 18th IEEE International Conference on Computer Communications and Networks (ICCCN), 2009, pp. 1-6.

[89] M .M .B. Tariq, M. Ammar, and E. Zegura. "Message Ferry Route Design for Sparse Ad Hoc Networks with Mobile Nodes", In Proceedings of 7th ACM International Symposium on Mobile ad hoc networking and computing (MobiHoc), 2006, pp. 37-48.

[90] W. Zhao, M. Ammar, and E. Zegura. "A Message Ferrying Approach for Data Delivery in Sparse Mobile Ad Hoc Networks," In Proceedings of 5th ACM international symposium on Mobile ad hoc networking and computing (MobiHoc), 2004, pp. 187-198.

[91] T. Spyropoulos, K. Psounis, and C. S. Raghavendra, "Efficient routing in intermittently connected mobile networks: the multiple-copy case," In IEEE/ACM Transactions on Networking, 16(1) (2008), pp. 77-90.

[92] F. Li and J. Wu, "LocalCom: a community-based epidemic forwarding scheme in disruption-tolerant networks," In Proceedings of 6th Annual IEEE Communications Society Conference on Sensor, Mesh and Ad Hoc Communications and Networks (SECON), 2009, pp. 1-9.

[93] F. Hou and X. Shen, "An adaptive forwarding scheme for message delivery over delay tolerant networks," In Proceedings of the IEEE Global Telecommunications Conference (GLOBECOM), 2009, pp. 1-5.

[94] P. Ramanathan and A. Singh, "Delay-differentiated gossiping in delay tolerant networks," In Proceedings IEEE International Conference on of the Communications (ICC), 2008, pp. 3291-3295.

[95] R. Subramanian and F. Fekri, "Throughput performance of network-coded multicast in an intermittently-connected network," In Proceedings of 8th International Symposium on Modeling and Optimization in Mobile, Ad Hoc and Wireless Networks (WiOpt), 2010, pp. 212-221.

[96] U. Lee, S. Y. Oh, W. K. Lee, and M. Gerla, "Relaycast: Scalable multicast routing in delay tolerant networks," In Proceedings of IEEE International Conference on Network Protocols (ICNP), 2008. pp. 218-227.

[97] P. Yang and M. C. Chuah, "Context-aware multicast routing scheme for disruption tolerant networks," In Proceedings of the 3rd ACM international workshop on Performance evaluation of wireless ad hoc, sensor and ubiquitous networks, 2006, pp. 66-73.

[98] G. Zyba, G. M. Voelker, S. Ioannidis, and C. Diot, "Dissemination in opportunistic mobile ad-hoc networks: The power of the crowd," In Proceedings of IEEE International Conference on Computer Communications (INFOCOM), 2011, pp. 1179-1187.

[99] M. Piorkowski, N. Sarafijanovic-Djukic, and M. Grossglauser, "A parsimonious model of mobile partitioned networks with clustering," In Proceedings of 1st International Communication Systems and Networks and Workshops, (COMSNETS), 2009, pp. 1-10.

[100] T. Henderson, D. Kotz, and I. Abyzov, "The changing usage of a mature campus-wide wireless network," In Computer Networks, 52 (14) (2008), pp. 2690-2712.

[101] Second Life, $<$ http://www.secondlife.com> [accessed on 8 March, 2015].

[102] M. Varvello, F. Picconi, C. Diot, and E. Biersack, "Is There Life in Second Life?," In ACM International Conference on Emerging Networking EXperiments and Technologies (CoNEXT), 2008.

[103] F. Neves dos Santos, B. Ertl, C. Barakat, T. Spyropoulos, and T. Turletti, "CEDO: Content-centric dissemination algorithm for delay-tolerant networks," In Proceedings of 16th ACM International conference on Modeling, Analysis and Simulation of Wireless and Mobile systems (MSWiM), 2013, pp. 377-386

[104] E. Talipov, Y. Chon, and H. Cha, "Content sharing over smart phone-based delay-tolerant networks," In IEEE Transactions on Mobile Computing, 12 (3) (2013), pp. 581-595.

[105] L. J. Chen, C. H. Yu, C. L. Tseng, H. H. Chu, and C. F. Chou, "A content-centric framework for effective data dissemination in opportunistic networks," In IEEE Journal on Selected Areas in Communications, 26 (5) (2008), pp. 761-772.

[106] L. J. Chen, C. H. Yu, T. Sun, Y. C. Chen, and H. H. Chu, "A hybrid routing approach for opportunistic networks," In Proceedings of ACM Special Interest Group on Data Communication (SIGCOMM) workshop on Challenged networks, 2006, pp. 213-220.

[107] O. Helgason and G. Karlsson, "Podnet: A system architecture for opportunistic content distribution," Technical report, Royal Institute of Technology (KTH) (February 2010). 
[108] J. W. Gao, G. Cao, A. Iyengar, and M. Srivatsa, "Supporting cooperative caching in disruption tolerant networks," In Proceedings of 31st International Conference on Distributed Computing Systems (ICDCS), 2011, pp. 151-161.

[109] W. Yu, H. Li, J. Li, L. Jin, and K. Yuan, “Transmission Scheduling Algorithm in DTN," In Communications and Network, 5 (3) (2013), pp. 255.

[110] B. H. Singh and S. A Lal, “Considerations and Open Issues in Delay Tolerant Network'S (DTNs) Security,” In Wireless Sensor Network, 2 (8) (2010) pp. 645.

[111] Y. Lu, X. Li, Y. T. Yu, and M. Gerla, “Information-centric delay-tolerant mobile ad-hoc networks," In IEEE Conference on Computer Communications Workshops (INFOCOM WKSHPS), 2014, pp. 428-433.

[112] X. Deng, J. Wang, Y. Liu, and L. Chang, "A social similarity-aware multicast routing protocol in delay tolerant networks," In International Journal of Simulation and Process Modelling, 8 (4) (2013), pp. 248-256. 